\title{
The X-ray properties of weak-lensing-selected galaxy clusters
}

\author{
P. A. Giles, ${ }^{1 \star}$ B. J. Maughan, ${ }^{1}$ T. Hamana,${ }^{2}$ S. Miyazaki, ${ }^{2}$ M. Birkinshaw, ${ }^{1}$ \\ R. S. Ellis ${ }^{3}$ and R. Massey ${ }^{4}$ \\ ${ }^{1}$ HH Wills Physics Laboratory, University of Bristol, Tyndall Avenue, Bristol BS8 1TL, UK \\ ${ }^{2}$ National Astronomical Observatory of Japan, Mitaka, Tokyo 181-8588, Japan \\ ${ }^{3}$ Astronomy Department, MS 249-17, California Institute of Technology, Pasadena, CA 91125, USA \\ ${ }^{4}$ Institute of Computational Cosmology, Durham University, South Road, Durham DH1 3LE, UK
}

Accepted 2014 December 12. Received 2014 November 12; in original form 2013 December 9

\begin{abstract}
We present the results of an X-ray follow-up campaign targeting 10 weak-lensing (WL)selected galaxy clusters from a Subaru WL survey. Archival Chandra data exist for two of the clusters, and we obtain dedicated observations of the remaining eight. The WL clusters appear to fit the same scaling relation between X-ray luminosity and temperature as X-ray-selected clusters. However, when we consider the luminosity-mass relation, the WL-selected clusters appear underluminous by a factor $2.1 \pm 0.5$ (or, alternatively, more massive by $1.7 \pm 0.3$ ), compared to X-ray-selected clusters with X-ray-based mass estimates. By considering the effects of the centroid offset, Eddington bias, and triaxiallity, this difference can be reconciled. We use X-ray imaging data to quantify the dynamical state of the clusters and found that one of the clusters appears dynamically relaxed, and two of the clusters host a cool core, consistent with Sunyaev-Zel'dovich-effect-selected clusters. This fraction is much lower than observed in X-ray-selected cluster samples.
\end{abstract}

Key words: galaxies: clusters: general - cosmology: observations - X-rays: galaxies: clusters.

\section{INTRODUCTION}

Clusters of galaxies are the largest gravitationally collapsed structures in the Universe. The study of their number density and growth from high-density perturbations in the early Universe offers insight into the underlying cosmology (e.g. Mantz et al. 2008; Vikhlinin et al. 2009b). However, in order to use clusters as a cosmological probe, three essential tools are required (Del Popolo, Costa \& Lanzafame 2010): (a) an efficient method to find clusters over a wide redshift range, (b) an observational method of determining the cluster mass, and (c) a method to compute the selection function or the survey volume in which clusters are found. A variety of observational techniques has been brought to bear on requirements $(\mathrm{a}-\mathrm{c}$ ), each with different strengths and weaknesses.

Early samples of clusters were based upon optical selection; however, these samples have traditionally suffered from projection effects and uncertainties in the optical richness-mass relation. Recently, the first clusters have been detected in blind surveys using the Sunyaev-Zel'dovich effect (SZE; Staniszewski et al. 2009; Vanderlinde et al. 2010; Planck Collaboration 2011a). This method holds much promise due to the redshift independence of the SZE (Birkinshaw 1999); however, the use of the SZE as a mass estimator remains largely untested. Until now, the most effective method of

^E-mail: P.Giles@ bristol.ac.uk building large, well-defined cluster samples has been via X-ray selection. The high X-ray luminosities of clusters makes it relatively easy to detect and study clusters out to high redshifts, and X-ray cluster studies have provided a means to impose tight constraints on cosmological parameters (e.g. Vikhlinin et al. 2009b; Mantz et al. 2010a).

A common weakness of the three techniques outlined above is that the clusters are selected based on the properties of their minority baryonic content. However, cosmological constraints from those clusters are based on their masses, dominated by dark matter. This gives rise to complications both in the estimation of cluster masses (which must be inferred from the observed baryon properties) and in the determination of sample selection functions. Incorporating selection functions in any cosmological model depends crucially on the form and scatter of the relationship between the observable used to detect the cluster, and the cluster mass.

In principle, these complications may be avoided by defining cluster samples through gravitational lensing, the most direct observational probe of cluster masses. The development of weak lensing (abbreviated WL throughout) techniques has enabled the detection of clusters via the distortions of background galaxy shapes leading to the construction of WL-selected cluster surveys. These include the Deep Lens Survey (DLS; Wittman et al. 2006) and our Subaru Weak Lensing Survey (Miyazaki et al. 2007), both $\approx 20 \mathrm{deg}^{2}$, and the large $\left(\sim 170 \mathrm{deg}^{2}\right)$ CFHT Legacy Survey (Shan et al. 2012). While WL techniques are free from assumptions about the relationship 
Table 1. Basic properties of the cluster sample. Columns: (1) = Source name; (2) = ObsID of the observation (4/5 digits Chandra, 10 digits XMM); (3) = Right ascension at J2000 of the WL peak (Hamana et al. 2009); (4) = Declination at J2000 of the WL peak (Hamana et al. 2009); (5) = Redshift of the cluster as determined from the Subaru spectroscopic follow-up (Hamana et al. 2009); (6) Cleaned exposure time; (7) Gives the total number of background-subtracted, core-excluded, cluster counts for the spectral analysis (with the SNR in brackets); (8) Refers to the analysis method used to determine the properties of the cluster (see Section 3). ${ }^{\dagger}$ Cluster observed with XMM.

\begin{tabular}{lccccccc}
\hline \multicolumn{1}{c}{ Cluster } & ObsID & RA & DEC & $z$ & Exposure (ks) & Analysis & Counts (sn) \\
\hline SLJ0225.7-0312 & 0553910201 & 022543.2 & -031236 & 0.1395 & 12 & Standard & $5124(55)$ \\
SLJ1647.7+3455 & 0652400401,12303 & 164747.5 & +345513 & 0.2592 & 13 & Standard & $1251(17)$ \\
SLJ0850.5+4512 & 12305 & 085031.7 & +451212 & 0.1935 & 29 & Standard & $167(3.8)$ \\
SLJ1000.7+0137 & $8022,8023,8555$ & 100045.5 & +013926 & 0.2166 & 98 & Standard & $2310(23)$ \\
SLJ1135.6+3009 & 12302 & 113538.4 & +300936 & 0.2078 & 11 & 'Alt'(c) & $71(3.2)$ \\
SLJ1204.4-0351 & 12304 & 120422.9 & -035055 & 0.2609 & 23 & Standard & $320(7.2)$ \\
SLJ1335.7+3731 & 12307 & 133545.6 & +373148 & 0.4070 & 27 & Standard & $138(5.2)$ \\
SLJ1337.7+3800 & 12306 & 133743.7 & +380057 & 0.1798 & 34 & 'Alt'(b) & $172(6.4)$ \\
SLJ1602.8+4335 & 12308 & 160252.8 & +433524 & 0.4155 & 42 & Standard & $717(12)$ \\
SLJ1634.1+5639 & 12309,13145 & 163412.0 & +563936 & 0.2377 & 48 & 'Alt'(a) & $164(3.0)$ \\
\hline
\end{tabular}

between baryonic and dark matter in clusters, they are susceptible to the possibility of projection of multiple structures along the line of sight (LOS), leading to overestimates of cluster masses or false detections. A recent study of clusters detected in the DLS found that five out of 12 cluster candidates appeared to be false detections (Starikova et al. 2014). However, they find that the scaling between velocity dispersion, $L_{\mathrm{X}}$ and $T_{\mathrm{X}}$, follow previously published scaling relations.

This paper aims to determine the X-ray properties of clusters detected via their WL signal. By constructing scaling relations based on the measured X-ray properties, we will determine whether the clusters follow simple scaling theory, which is a key ingredient in the determination of cosmological parameters. One of the most important measurements for use in cosmological studies is that of the cluster' mass. Much work has been done to determine cluster masses from WL observations, however this is primarily based on X-ray-selected cluster samples (e.g. Hoekstra 2007; Okabe et al. 2010b; Jee et al. 2011; Hoekstra et al. 2012; Israel et al. 2012; Mahdavi et al. 2013). We will compare the scaling relation between the X-ray luminosity and WL mass from WL-selected and X-rayselected samples. The derivation of the WL cluster mass assumes that the clusters follow spherical symmetry. We investigate this assumption and determine the dynamical state and cool-core (CC) fraction of WL-selected clusters.

The outline of this paper is as follows. In Section 2, we discuss the sample selection and data reduction. The derivation of the cluster properties is given in Section 3. In Section 4, we present the results of our X-ray analysis and derive scaling relations. Our discussions and conclusions are presented in Sections 5 and 6, respectively. Throughout this paper, we assume a cosmology with $\Omega_{\mathrm{M}}=0.3$, $\Omega_{\Lambda}=0.7$, and $H_{0}=70 \mathrm{~km} \mathrm{~s}^{-1} \mathrm{Mpc}^{-1}$.

\section{SAMPLE AND DATA REDUCTION}

Our clusters were selected from the Subaru WL survey of Miyazaki et al. (2007). Briefly, 100 significant shear signals, with a threshold signal-to-noise ratio $(\mathrm{SNR})>3.69$, were detected in a $16.7 \mathrm{deg}^{2}$ area (after avoiding areas close to bright stars and field boundaries). A preliminary comparison in Miyazaki et al. (2007) to the $X M M$-LSS field survey found 80 per cent of shear signals had a confirmed cluster counterpart. The initial WL survey was designed to detect clusters out to $z \approx 0.5$, for a halo of mass $M_{\mathrm{vir}} \sim 10^{14} \mathrm{M}_{\odot}$
(Hamana, Takada \& Yoshida 2004). The sample that we use in this work was constructed based upon confirmed cluster identification from spectroscopic follow-up (Hamana et al. 2009). The clusters selected for spectroscopic follow-up were selected from both the peak gravitational-lensing density, $\kappa$, and a visual inspection of the optical images. For 36 WL cluster candidates detected in Miyazaki et al. (2007), 15-32 galaxy redshifts were obtained per cluster, with $28(\approx 80$ per cent) candidates securely identified as clusters. 10 of these clusters (within the redshift range $0.14 \leq z \leq 0.42$ ) were defined as a 'clean' subset, whose velocity dispersion could be evaluated from at least 12 spectroscopic member galaxies, and whose WL mass estimates are not affected by a neighbouring system or field boundary. A summary of the 10 clusters investigated in this paper is given in Table 1. For eight of the clusters in the sample, we obtained dedicated Chandra pointings. The other two clusters are SLJ1000.7, which was observed in the COSMOS field with Chandra, and SLJ0225.7, was observed in the XMM_LSS_13 field with $X M M$. We note however that the Chandra observation of SLJ1647.7 produced low-quality data. We therefore chose to use an archived XMM observation for the analysis of this cluster.

For the eight clusters analysed using Chandra observations, we used the CIAO ${ }^{1} 4.4$ software package with CALDB $^{2}$ version 4.4 .7 and followed standard reduction methods. Since all observations were telemetered in VFAINT mode, additional background screening was applied. ${ }^{3}$ We inspected background light curves of the observations following the recommendations given in Markevitch et al. (2003), to search for possible background fluctuations. None of the observations were affected by periods of background flaring.

In order to take the background into account, we follow the method described in Vikhlinin et al. (2006). Briefly, Chandra blanksky backgrounds were obtained, processed identically to the cluster, and reprojected on to the sky to match the cluster observation. We then renormalized the background in the 9.5-12 keV band, where the Chandra effective area is nearly zero and the observed flux is due entirely to the particle background events. Finally, to take into account differing contributions from the soft X-ray background, the spectra were subtracted and residuals were modelled in the 0.4-1 keV band using an unabsorbed APEC thermal plasma model

\footnotetext{
${ }^{1}$ See http://cxc.harvard.edu/ciao/.

${ }^{2}$ See http://cxc.harvard.edu/caldb/.

${ }^{3}$ See http://cxc.harvard.edu/ciao/why/aciscleanvf.html.
} 
(Smith et al. 2001) with a temperature of $0.18 \mathrm{keV}$. This model was included in the spectral fitting for the cluster analysis.

The clusters SLJ0225.7 and SLJ1647 were observed by XMM. We used the Science Analysis Software (SAS) version 12.0.1 and the most recent calibration products as of 2013 October for the analysis of these clusters.

Table 1 lists the total cleaned exposure times for each cluster.

\section{CLUSTER PROPERTIES}

In this section, we outline the methods used to determine the cluster properties of our sample. Where possible we use the method outlined in Section 3.1; however, for clusters with low-SNR data, the properties are determined using one of the methods described in Section 3.2. Table 1 lists the analysis method used for each cluster.

\subsection{The 'standard' method}

To determine the cluster properties of our sample, we followed the procedures outlined in Maughan et al. (2012). Briefly, cluster spectra were extracted and fits performed in the $0.6-9.0 \mathrm{keV}$ (0.4-7.0 keV for the XMM clusters to avoid instrumental lines) band with an absorbed APEC model, with the absorbing column fixed at the Galactic value (Kalberla et al. 2005). Due to the low SNR of three of the clusters (see Section 3.3), the abundance was fixed at $0.3 \mathrm{Z}_{\odot}$ throughout this analysis. We determine the gas density profile for each cluster by converting the observed surface brightness profile (constructed in the $0.7-2.0 \mathrm{keV}$ band, and binned to contain at least 10 counts per bin) into a projected emissivity profile, which is modelled by projecting a density model along the LOS. We use the model of Vikhlinin et al. (2006, see that work for parameter definitions):

$n_{\mathrm{p}} n_{\mathrm{e}}=\frac{n_{0}^{2}\left(r / r_{\mathrm{c}}\right)^{-\alpha}}{\left(1+r^{2} / r_{\mathrm{c}}^{2}\right)^{3 \beta-\alpha / 2}} \times\left(1+r^{\gamma} / r_{s}^{\gamma}\right)^{-\epsilon / \gamma}$.

The same parameter constraints were employed as in Vikhlinin et al. (2006), i.e. $\gamma$ is fixed at 3 and $\epsilon<5$ to exclude non-physical sharp density peaks. Gas masses were determined from Monte Carlo realizations of the projected emissivity profile based on the bestfitting projected model to the original data. Analysis of the $X M M$ clusters followed the same method outlined here, but did not include corrections for the point spread function (PSF). However, the radial profiles used to generate the emissivity profiles were binned to a width of 15 arcsec, limiting the effect of the XMM PSF.

The cluster temperature, gas mass, and $r_{500}$ (the radius at which the enclosed density of the cluster becomes 500 times the critical density at the cluster's redshift) were determined through an iterative process. We extract a spectrum from within an estimate of $r_{500}$ (with the central 15 per cent excluded), integrate a gas density profile (see Maughan et al. 2008) to determine the gas mass, and then calculate a value for $Y_{\mathrm{X}}$ (the product of the temperature and gas mass; Kravtsov, Vikhlinin \& Nagai 2006). A new $r_{500}$ was then estimated from the $Y_{X}-\mathrm{M}$ scaling relation of Vikhlinin et al. (2009a):

$M_{500}=E(z)^{-2 / 5} A_{\mathrm{YM}}\left(\frac{Y_{\mathrm{X}}}{3 \times 10^{14} \mathrm{M}_{\odot} \mathrm{keV}}\right)^{B_{\mathrm{YM}}}$,

with $A_{\mathrm{YM}}=5.77 \times 10^{14} h^{1 / 2} \mathrm{M}_{\odot}$ and $B_{\mathrm{YM}}=0.57$. Here, $M_{500}$ is the mass within $r_{500}$, where $r_{500}$ is defined as $r_{500}=\left(3 M_{500} / 4 \pi 500 \rho_{\mathrm{c}}(z)\right)^{1 / 3}$, where $\rho_{\mathrm{c}}(z)$ is the critical density of the Universe at the cluster redshift. The $Y_{X}-M_{500}$ relation assumes self-similar evolution (corrected by $E(z)^{-2 / 5}$ ), where
$E(z)=\sqrt{\Omega_{\mathrm{M}}(1+z)^{3}+\left(1-\Omega_{\mathrm{M}}-\Omega_{\Lambda}\right)(1+z)^{2}+\Omega_{\Lambda}}$ (justified by Maughan 2007). An initial temperature of $2 \mathrm{keV}$ was used to determine an initial $r_{500}$ and then this process was repeated until $r_{500}$ converged to within 1 per cent. The luminosity and temperature were measured from spectra extracted within $r_{500}$ both with and without the central 15 per cent of $r_{500}$ excluded. Throughout, we define $L_{\mathrm{X}}$ and $k T$ as core-excluded cluster properties, and $L_{\mathrm{X}, \mathrm{c}}$ and $k T_{\mathrm{c}}$ as core included properties. All luminosities quoted throughout are bolometric unless otherwise stated.

\subsection{The 'alternative' methods}

Due to the low SNR of the clusters resulting from the clusters being less luminous than expected based on the WL mass (used when planning the observations), three of the clusters in our sample could not be analysed using the method described in Section 3.1. We therefore used a variety of methods in order to obtain the most accurate properties for these low-SNR clusters. In order of preference, these were as follows.

(a) When the $Y_{\mathrm{X}}$-mass relation could not be used to determine $r_{500}$, we used the $r_{500}-T$ relation given in Vikhlinin et al. (2006) to deduce $r_{500}$, and followed the iterative process detained in Section 3.1 to determine the cluster properties within $r_{500}$. We note that for consistency with the assumed $r_{500}-T$ relation, the central $70 \mathrm{kpc}$ is excluded (instead of $0.15 r_{500}$ as in Section 3.1). Generally, the $70 \mathrm{kpc}$ region is smaller than the $0.15 r_{500}$ region, and hence increases the SNR. Therefore, this method can be used to determine $r_{500}$ for lower SNR clusters.

(b) Extracting a spectrum within a region to give the highest SNR for the cluster and determining the properties within this region. Again, the central $70 \mathrm{kpc}$ is excluded for this analysis for the same reason as in method (a). $r_{500}$ was then estimated using the $r_{500}-$ $T$ relation of Vikhlinin et al. (2006) and $L_{\mathrm{X}}$ was extrapolated to the radius by integrating under a $\beta$-profile with $r_{c}=150 \mathrm{kpc}$ and $\beta=0.667$.

(c) Using PIMMS ${ }^{4}$ to determine the cluster luminosity from the count rate of the cluster observation when no spectroscopic analysis could be performed. A global temperature of $2 \mathrm{keV}$ was assumed for the cluster, and the count rate determined in an $r_{500}$ determined from the $r_{500}-T$ relation of Vikhlinin et al. (2006).

In order to test for any systematic differences in our $r_{500}$ measurements due to using the 'alternative' method, we computed $r_{500}$ for all clusters by assuming an isothermal $\beta$-model of the form

$\rho(r)=\rho_{0}\left[1+\left(\frac{r}{r_{\mathrm{c}}}\right)^{2}\right]^{-3 \beta / 2}$,

where we assume $r_{\mathrm{c}}=150 \mathrm{kpc}$ and $\beta=0.667$ for all clusters. The central density was calculated using the normalization of the APEC model used in the spectral fits. We found that the ratio of the $r_{500} \mathrm{~s}$ calculated using the $\beta$-model, and the methods described above, is consistent to about 1 per cent. Any systematics introduced by using the 'Alternative' method for the low-SNR clusters are negligible in our later analysis.

\subsection{Notes on individual clusters}

Notes on the WL detections of the individual clusters can be found in Hamana et al. (2009). In this section, we note any peculiarities

${ }^{4}$ http://cxc.harvard.edu/toolkit/pimms.jsp 
or points of interest for observations in which we departed from analysis process described in Section 3.1. All five observations that were analysed using the method described in Section 3.2 are discussed below.

\subsubsection{SLJ1000.7+0137}

The cluster SLJ1000.7+0137 had three archival Chandra observations taken in the COSMOS-6 field. An image of the Chandra observation is shown in Fig. A3(a). The three individual observations were analysed separately as described in Section 3.1. The data were then combined for certain stages of the analysis. Source, background, and exposure maps were projected on to common coordinated systems and summed. Source and background spectra were extracted for individual observations and fit simultaneously with the temperatures of the hot APEC components tied together.

\subsubsection{SLJ1135.6+3009}

In order to calculate the luminosity of this cluster, we followed method (c) outlined in Section 3.2. We determined the number of counts in a region centred on the location of the brightest cluster Galaxy (BCG, see Fig. A4 with the BCG highlighted by the black circle), subtracting the number of counts from the same region in a blank-sky background obtained for the observation, scaled by the ratio of the source and background exposure times. The net number of counts obtained was $71 \pm 22$ (significant at the $3.2 \sigma$ level), in the $0.7-2.0 \mathrm{keV}$ band. We calculated a bolometric luminosity of $L_{\mathrm{X}, \mathrm{c}}=(1.28 \pm 0.40) \times 10^{43} \mathrm{ergs} \mathrm{s}^{-1}$ for the cluster, with the errors derived assuming Poisson statistics.

\subsubsection{SLJ1337.7+3800}

To determine the cluster properties we used method (b) in Section 3.2, extracting spectra within a radius [70-215]kpc. The net number of spectral counts, corrected for background, was then 130 (in the 0.6-9.0 keV band, with SNR = 5.3). We find a temperature of $k T=1.63_{-0.28}^{+0.68} \mathrm{keV}$, corresponding to an $r_{500}$ of $574 \mathrm{kpc}$. We derive an extrapolated bolometric luminosity of the cluster of $L_{\mathrm{X}}=(6.58 \pm 1.31) \times 10^{42} \mathrm{ergs} \mathrm{s}^{-1}$. This analysis was repeated including the core (obtaining 172 net spectral counts in the $0.6-9.0 \mathrm{keV}$ band, with $\mathrm{SNR}=6.45$ ). We determined a temperature of $k T_{\mathrm{c}}=1.63_{-0.23}^{+0.47} \mathrm{keV}$ and an extrapolated bolometric luminosity of $L_{\mathrm{X}, \mathrm{c}}=(8.56 \pm 1.21) \times 10^{42} \mathrm{ergs} \mathrm{s}^{-1}$.

\subsubsection{SLJ1634.1+5639}

Due to observational constraints, the exposure was split into two observations. Therefore, the analysis of the cluster was performed as described in Section 3.3.1. The properties of this cluster were obtained following the process outlined in method (a) in Section 3.2. We find a temperature of $k T=1.37_{-0.44}^{+0.80} \mathrm{keV}$ and $L_{\mathrm{X}}=(4.17 \pm 1.67) \times 10^{42} \mathrm{ergs} \mathrm{s}^{-1}$. Including the core of the cluster, we found a temperature and luminosity of $k T_{\mathrm{c}}=1.72_{-0.43}^{+0.95} \mathrm{keV}$ and $L_{\mathrm{X}, \mathrm{c}}=(6.78 \pm 2.59) \times 10^{42} \mathrm{ergs} \mathrm{s}^{-1}$.

Substructure in SLJ1634 appears likely on the basis of the locations of the galaxies, as marked in Fig. A9, which shows a split in the population of galaxies in the northern and southern region of the cluster. To check for the reality (or otherwise) of this structure, a tree analysis of the locations of the galaxies in 3D (position, velocity, redshift) space was undertaken. The results suggest a composite structure for the cluster, with the galaxies separated into three separate groups. The galaxies in Fig. A9 are colour coded with respect to each group they are associated. Group A (magenta squares), B (black squares), and C (green squares) have a redshift of 0.231 , 0.238 , and 0.242 , respectively. This composite structure may explain the large offset in the luminosity-mass relation for SLJ1634 when the WL mass is calculated centred on the X-ray peak (see Fig. 5).

\subsubsection{SLJ1647.7+3455}

Although a dedicated Chandra observation was obtained for the cluster SLJ1647.7+3455, due to the non-detection of the cluster, no useful information could be determined. We therefore used an archival $X M M$ observation to determine the properties of the cluster.

\section{RESULTS}

In this section, we present the results of our analysis of our 10 WL-selected clusters. We derive various scaling relations for these clusters and compare to published results. The measured properties of the clusters are given in Table 2, along with the WL mass determined in Hamana et al. (2009) converted to $H_{0}=70 \mathrm{~km} \mathrm{~s}^{-1} \mathrm{Mpc}^{-1}$. We note two updates to the derivation of the WL masses given in Hamana et al. (2009), and those presented in this work. We use the halo mass-concentration relation of Duffy et al. (2008), and the modified NFW profile given in Oguri \& Hamana (2011)

\subsection{The luminosity-temperature relation}

Here, we derive the X-ray luminosity-temperature (LT) relation for our clusters, omitting SLJ1135 for which a temperature could not be obtained. Fig. 1 shows the $L_{\mathrm{X}, \mathrm{c}}-k T_{\mathrm{c}}$ relation for our clusters (red triangles) compared with the LT relation given in Maughan et al. (2012, blue open circles). The Maughan et al. (2012) sample of clusters was selected from all available Chandra pointings as of 2006 November, correlated with the NASA/IPAC Extragalctic Database (NED) and observations with a galaxy group or cluster listed in NED within 10 arcmin of the Chandra aim point were kept. Further criteria included observations only carried out with the ACIS-I detector, a galaxy group/cluster as the target, and a published redshift $>0.1$. As the Maughan et al. (2012) sample of clusters covers a wide redshift range $(0.1<z<1.3)$, the luminosities of the clusters were corrected for the expected self-similar evolution, given by $L_{\mathrm{X}} \times E(z)^{-1}$. The same correction was also applied to our data.

A power law of the form $L_{X}=E(z)^{2} L_{0}\left(k T / k T_{0}\right)^{B_{\mathrm{LT}}}$ was fit to the data using the BCES orthogonal regression in log space (Akritas \& Bershady 1996) with $k T_{0}$ set at $2 \mathrm{keV}$. From our fit, we find a normalization of $L_{0}=(2.44 \pm 0.51) \times 10^{43} \mathrm{ergs} \mathrm{s}^{-1}$ and a slope of $B_{\mathrm{LT}}=2.63 \pm 0.69$. The slope and normalization are consistent with the fit to the Maughan et al. (2012) sample, who find a normalization of $L_{0, \mathrm{M} 12}=(2.40 \pm 0.78) \times 10^{43} \mathrm{ergs} \mathrm{s}^{-1}$ at $2 \mathrm{keV}$ and a slope of $B_{\mathrm{LT}, \mathrm{M} 12}=3.63 \pm 0.27$ (see Fig. 1, blue solid line). We conclude that the X-ray properties of the WL-selected clusters scale consistently with X-ray-selected clusters in the LT plane. 
Table 2. Derived X-ray properties of the clusters with WL mass estimates (see Section 4). Clusters in the top part of the table were analysed from XMM observations, in the bottom part from Chandra observations.

\begin{tabular}{cccccccc}
\hline \multicolumn{1}{c}{ Cluster } & $E(z)$ & $\begin{array}{c}L_{\mathrm{X}} \\
\left(\times 10^{43} \mathrm{ergs} \mathrm{s}^{-1}\right)\end{array}$ & $\begin{array}{c}L_{\mathrm{X}, \mathrm{c}} \\
\left(\times 10^{43} \mathrm{ergs} \mathrm{s}^{-1}\right)\end{array}$ & $\begin{array}{c}L_{0.1-2.4 \mathrm{keV}, \mathrm{X}, \mathrm{c}} \\
\left(\times 10^{43} \mathrm{ergs} \mathrm{s}^{-1}\right)\end{array}$ & $\begin{array}{c}k T \\
(\mathrm{keV})\end{array}$ & $\begin{array}{c}k T_{\mathrm{c}} \\
(\mathrm{keV})\end{array}$ & $\begin{array}{c}M_{500}^{\mathrm{WL}} \\
\left(\times 10^{14} \mathrm{M}_{\odot}\right)\end{array}$ \\
\hline SLJ0225.7-0312 & 1.070 & $7.31 \pm 0.19$ & $7.90 \pm 0.19$ & $4.16 \pm 0.10$ & $2.93_{-0.19}^{+0.19}$ & $3.00_{-0.17}^{+0.18}$ & $1.97_{-0.47}^{+0.47}$ \\
SLJ1647.7+3455 & 1.140 & $1.38 \pm 0.12$ & $1.57 \pm 0.12$ & $1.31 \pm 0.09$ & $1.49_{-0.11}^{+0.11}$ & $1.58_{-0.10}^{+0.09}$ & $2.00_{-0.79}^{+0.67}$ \\
SLJ0850.5+4512 & 1.100 & $0.78 \pm 0.14$ & $0.92 \pm 0.13$ & $0.83 \pm 0.13$ & $1.21_{-0.16}^{+0.22}$ & $1.16_{-0.15}^{+0.14}$ & $1.09_{-0.43}^{+0.39}$ \\
SLJ1000.7+0137 & 1.114 & $4.04 \pm 0.17$ & $5.62 \pm 0.19$ & $3.80 \pm 0.17$ & $2.43_{-0.29}^{+0.29}$ & $2.61_{-0.23}^{+0.41}$ & $2.39_{-0.53}^{+0.46}$ \\
SLJ1135.6+3009 & 1.108 & - & $1.28 \pm 0.40$ & $1.08 \pm 0.26$ & - & - & $2.49_{-0.56}^{+0.50}$ \\
SLJ1204.4-0351 & 1.141 & $3.43 \pm 0.35$ & $3.97 \pm 0.38$ & $2.92 \pm 0.28$ & $2.16_{-0.37}^{+0.49}$ & $2.16_{-0.22}^{+0.59}$ & $1.20_{-0.60}^{+0.50}$ \\
SLJ1335.7+3731 & 1.239 & $3.10 \pm 0.71$ & $3.99 \pm 0.69$ & $3.35 \pm 0.68$ & $1.39_{-0.37}^{+0.63}$ & $1.78_{-0.47}^{+0.79}$ & $2.79_{-1.01}^{+0.90}$ \\
SLJ1337.7+3800 & 1.092 & $0.66 \pm 0.13$ & $0.86 \pm 0.12$ & $0.37 \pm 0.05$ & $1.63_{-0.28}^{+0.68}$ & $1.63_{-0.23}^{+0.47}$ & $1.24_{-0.39}^{+0.36}$ \\
SLJ1602.8+4335 & 1.245 & $12.7 \pm 1.14$ & $16.4 \pm 1.22$ & $8.50 \pm 0.54$ & $3.91_{-0.72}^{+1.72}$ & $4.45_{-1.12}^{+1.29}$ & $2.66_{-0.71}^{+0.69}$ \\
SLJ1634.1+5639 & 1.126 & $0.63 \pm 0.22$ & $0.68 \pm 0.26$ & $0.49 \pm 0.15$ & $1.37_{-0.23}^{+0.77}$ & $1.42_{-0.23}^{+0.83}$ & $0.87_{-0.49}^{+0.39}$ \\
\hline
\end{tabular}

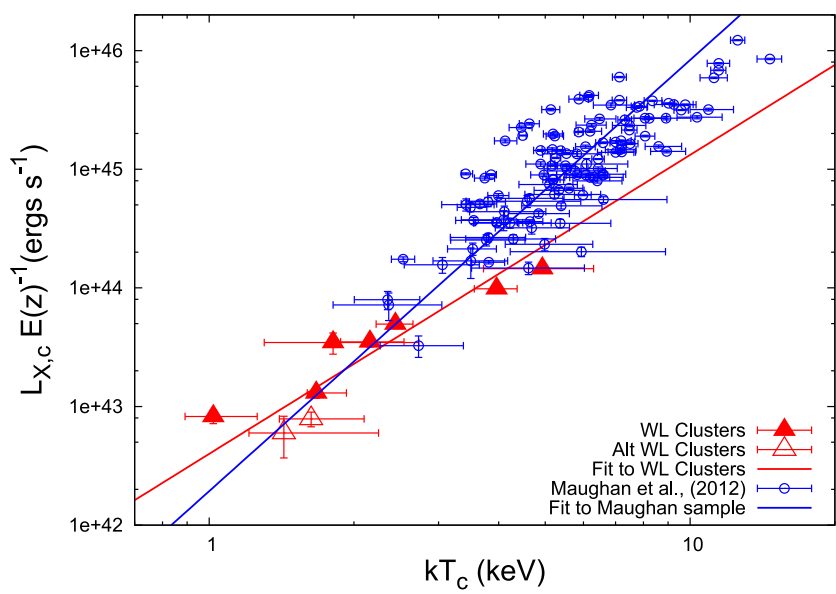

Figure 1. Figure showing the core-included luminosity-temperature $\left(L_{\mathrm{X}, \mathrm{c}}-k T_{\mathrm{c}}\right)$ relation for the WL-selected clusters (red triangles. Low-SNR clusters analysed using the 'Alternative' method are given by open triangles). For comparison, we overplot the $L_{\mathrm{X}, \mathrm{c}}-k T_{\mathrm{c}}$ relation of a sample of 114 X-ray-selected clusters of Maughan et al. (2012, blue open circles).

\subsection{The luminosity-mass relation}

\subsubsection{Properties derived within a WL $\mathrm{r}_{500}$}

Here, we derive the luminosity-mass (LM) relation for our clusters, with the masses estimated from the WL analysis presented in Hamana et al. (2009), updated as described in Section 4. Due to the WL selection of these clusters, we first derive the LM relation for the properties derived within the WL defined $r_{500}\left(r_{500}^{\mathrm{WL}}\right)$. However, due to the low SNR of three of the clusters, directly measuring properties within $r_{500}^{\mathrm{WL}}$ is not possible. We therefore extrapolate the $\mathrm{X}$-ray luminosities, defined within an X-ray $r_{500}\left(r_{500}^{\mathrm{X}}\right)$, out to $r_{500}^{\mathrm{WL}}$ using the method outlined in Section 3.2(b). Fig. 2 shows the LM relation for our WL clusters (given by the red triangles), with the properties derived within $r_{500}^{\mathrm{WL}}$. We compared to the X-ray-selected cluster sample presented in Mahdavi et al. (2013, hereafter M13, pink circles in Fig. 2), who present an X-ray and optical study of $50 \mathrm{X}$-ray-selected clusters in the redshift range $0.15<z<0.55$. Due to the availability of both WL masses (using data from the Canada-France-Hawaii Telescope, CFHT), and X-ray properties

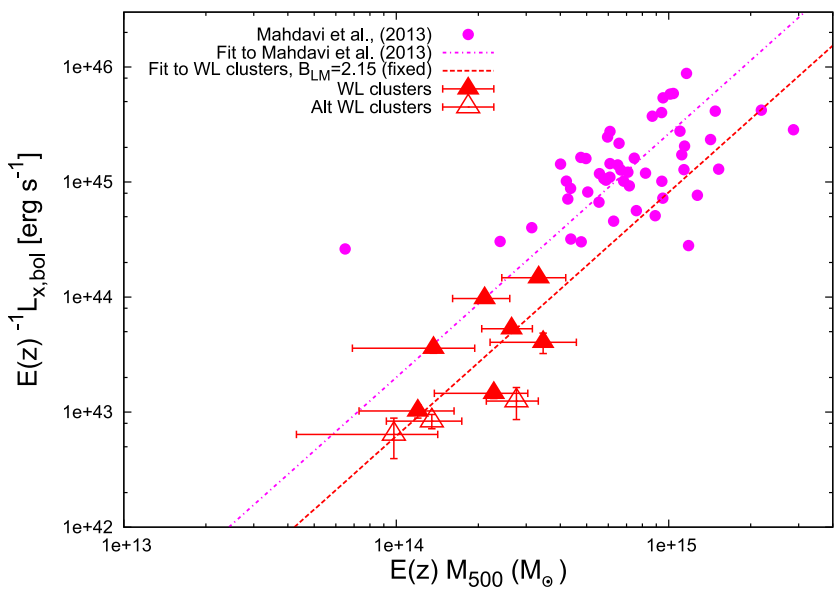

Figure 2. The LM relation for our WL-selected clusters (red triangles, clusters analysed using the 'Alternative' method are given by open triangles), with the properties derived within $r_{500}^{\mathrm{WL}}$. A power-law fit (see Section 4.2) to the clusters is given by the dashed red line. For comparison, we plot the data of the X-ray-selected clusters given in Mahdavi et al. (2013, pink circles), and the corresponding fit (pink dash-dotted line).

within $r_{500}^{\mathrm{WL}}, 5$ this sample is an ideal comparison. We note that there has been a recent erratum for this work (Mahdavi et al. 2014), which is taken into account in this comparison.

A power law of the form $E(z)^{-1} L_{\mathrm{X}}=L_{0}\left(E(z) M / M_{0}\right)^{B_{\mathrm{LM}}}$ was fit to the WL clusters in log space (as in Section 4.1), with $M_{0}$ set at $2 \times 10^{14} \mathrm{M}_{\odot}$. Due to the low quality of the data and the small number of data points, we fixed the slope $\left(B_{\mathrm{LM}}\right)$ of the relation at the best-fitting value found when fitting the M13 cluster sample, where we find $\mathrm{B}_{\mathrm{M} 13, \mathrm{LM}}=2.15 \pm 0.82$. Since the slope is fixed, we use a $\chi^{2}$ fit for the value of $L_{0}$, which we find to be $L_{0}=(2.7 \pm 0.5) \times 10^{43} \mathrm{erg} \mathrm{s}^{-1}$, for a mass of $2 \times 10^{14} \mathrm{M}_{\odot}$. The fits to the WL sample and the M13 sample are given by the red dashed line and pink dott-dashed line, respectively, in Fig. 2. We find a clear offset in the normalization of the fits to the two clusters samples, implying either our clusters are underluminous by $3.2 \pm 0.9$ compared to M13, or they are overmassive by a factor $1.7 \pm 0.2$. However, due to the consistency of the LT relation (see Fig. 1) with $\mathrm{X}$-ray-selected clusters, the discrepancy is more likely be associated

${ }^{5}$ downloaded from http://sfstar.sfsu.edu/cccp/. 


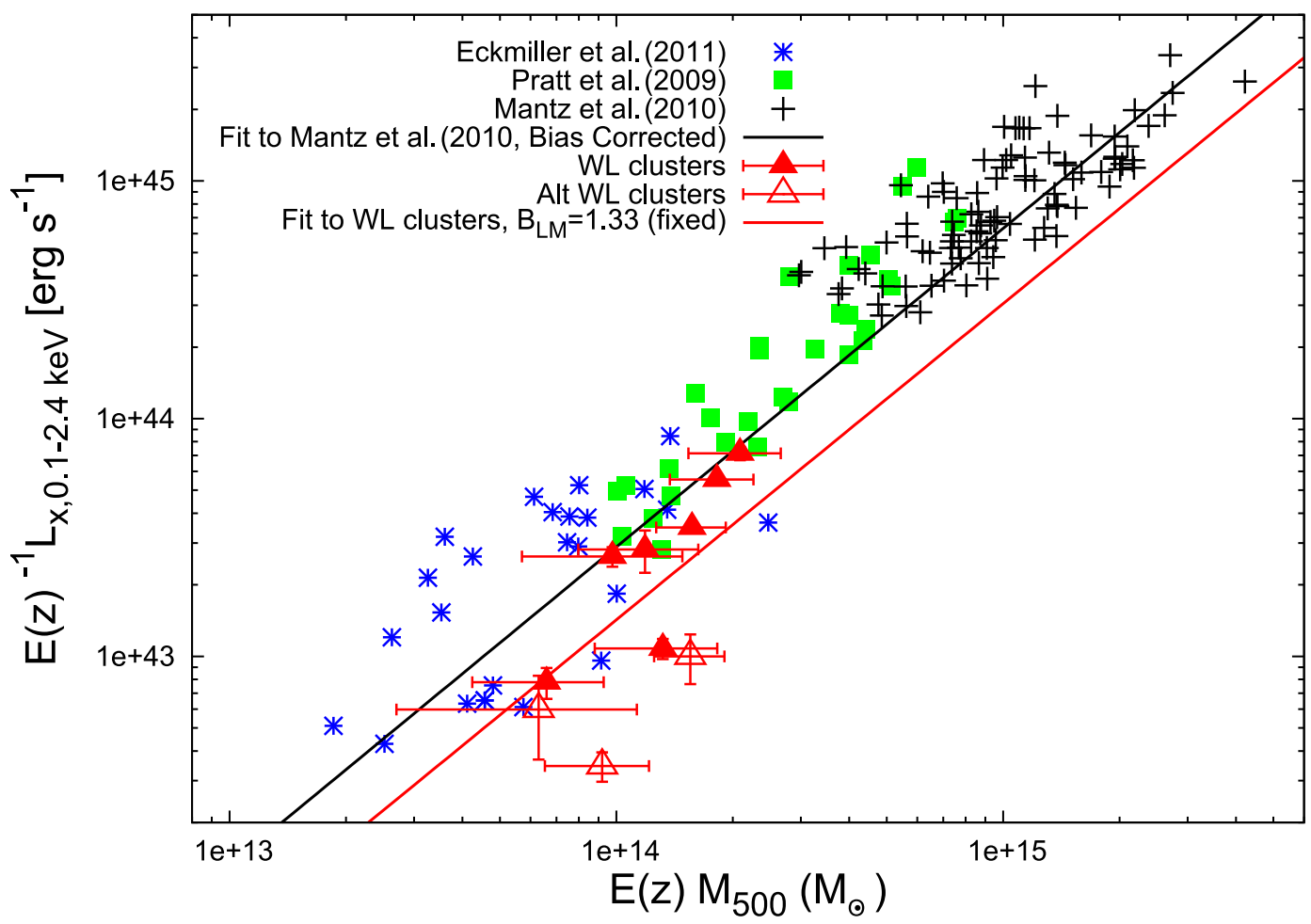

Figure 3. The LM relation for our WL-selected clusters (red triangles. Low-SNR clusters analysed using the 'Alternative' methods are given by open triangles), with WL masses derived within $r_{500}^{\mathrm{X}}$. For comparison, we plot the data of X-ray-selected clusters given in Mantz et al. (2010a, black crosses), and the corresponding fit taking into account selection effects (black line). Further comparisons are made to the REXCESS cluster sample (Pratt et al. 2009, green squares) and galaxy group sample of Eckmiller, Hudson \& Reiprich (2011, blue stars).

with a bias in the mass. Not only this, but X-ray-selected clusters suffer from biases, which must be minimized in this comparison. We therefore re-derived the WL masses of our clusters within $r_{500}^{\mathrm{X}}$, and compared our value to published work where the selection effects have been fully accounted for when modelling the LM relation.

\subsubsection{Properties derived within an $X$-ray $\mathrm{r}_{500}$}

In order to enable comparisons with the LM relation of clusters across a broader mass range, we henceforth use X-ray luminosities and WL masses measured in an $r_{500}$ based on the X-ray properties of the cluster (see Section 3). This allows us to make comparisons with large samples of X-ray-selected clusters with X-ray-based masses and luminosities measured in a consistent $\mathrm{X}$-ray based $r_{500}$.

Fig. 3 shows the LM relation for our clusters (red triangles), with properties derived within $r_{500}^{\mathrm{X}}$, compared to the data given in Mantz et al. (2010b, black crosses). Their clusters are taken from the ROSAT Brightest Clusters Sample (Ebeling et al. 1998), the ROSAT-ESO Flux-Limited X-ray Sample (Böhringer et al. 2004), and the bright subsample of the Massive Cluster Survey (Ebeling et al. 2010), all $z<0.5$. We note that Mantz et al. (2010b) used the gas mass as a proxy for the total cluster mass. The luminosities in Mantz et al. (2010b) are calculated in the $0.1-2.4 \mathrm{keV}$ band (cluster rest frame), we therefore calculated the luminosities of the WL clusters in the same band for the purpose of this comparison.

Since we wish to compare WL- and X-ray-selected clusters, we need to minimize any selection effects in the X-ray comparison sample. X-ray-selected cluster samples suffer from both Malmquist and Eddington biases. Malmquist bias refers to the effect that a larger survey volume finds more luminous objects for a given flux limit, and Eddington bias refers to the differential scattering of objects across the detection threshold of a survey due to the slope of the mass function. The analysis presented in Mantz et al. (2010b) takes both biases fully into account, making it an ideal comparison for our sample. We also note that the correction for the $E(z)$ factor in Mantz et al. (2010b) for the LM relation was based upon the bestfitting cosmology found in Mantz et al. (2010a). For the purposes of the LM relation comparison, the $E(z)$ factor for each cluster is also calculated using the Mantz et al. (2010a) cosmology. On average, $E(z)$ changes by $\approx 3$ per cent.

A power law of the form $E(z)^{-1} L_{\mathrm{X}}=L_{0}\left(E(z) M / M_{0}\right)^{B_{\mathrm{LM}}}$ was fit to the WL clusters as in Section 4.2.1. We again fixed the slope $\left(B_{\mathrm{LM}}\right)$ at the self-similar expectation value of 1.33. A $\chi^{2}$ fit gives $L_{0}=(3.6 \pm 0.5) \times 10^{43} \mathrm{ergs} \mathrm{s}^{-1}$ (red solid line in Fig. 3), for a mass $2 \times 10^{14} \mathrm{M}_{\odot}$. The fit from Mantz et al. (2010a) is represented by the solid black line. The low normalization compared to the data demonstrates the sizes of the Malmquist and Eddington biases. The Mantz et al. (2010a) LM relation at the same mass gives $L_{0}=7.3 \times 10^{43} \mathrm{ergs} \mathrm{s}^{-1}$. Compared with X-ray-selected clusters, the WL-selected sample shows X-ray luminosities are lower at a given mass by a factor $2.1 \pm 0.5$. Conversely, $M_{500}^{\mathrm{WL}}$ is higher for a given luminosity by a factor $1.7 \pm 0.3$. We discuss the nature of this offset in the LM relation in Section 5.

The main drawback in the comparison with the Mantz et al. (2010a) sample of clusters, and indeed the comparison with M13 above, is the mass range covered by the respective cluster samples. The Mantz et al. (2010a) sample of clusters have masses $M_{500} \geq 3 \times 10^{14} \mathrm{M}_{\odot}$, whereas our WL-selected sample are all $<2.7 \times 10^{14} \mathrm{M}_{\odot}$. To ensure the mass range of the comparison 
sample is not playing a role in the large observed offset, we compare to a sample of clusters probing the lower mass end and a sample of galaxy groups. We use the REXCESS (Böhringer et al. 2007) sample of clusters studied in Pratt et al. (2009) which span the mass range $\approx 10^{14}-10^{15} \mathrm{M}_{\odot}$, and the sample of galaxy groups presented in Eckmiller et al. (2011) spanning the mass range $\approx(0.05-2) \times 10^{14} \mathrm{M}_{\odot}$. The masses of the Pratt et al. (2009) clusters were calculated using the $Y_{\mathrm{X}}$-mass relation given in Arnaud, Pointecouteau \& Pratt (2007), and the masses of the Eckmiller et al. (2011) groups were calculated by assuming hydrostatic equilibrium and spherical symmetry (see section 3.4 in Eckmiller et al. 2011). In both cases, the luminosities are calculated in the $0.1-2.4 \mathrm{keV}$ band (cluster rest frame). We plot the clusters on the LM relation in Fig. 3, with the Pratt et al. (2009) clusters given by the green squares and the Eckmiller et al. (2011) groups given by the blue stars. A visual inspection shows that all the X-ray-selected comparison groups lie on approximately the same LM relation. Although the Pratt et al. (2009) and Eckmiller et al. (2011) samples appear to lie slightly above the Mantz et al. (2010a) relation, this is consistent with a similar level of bias as in the Mantz et al. (2010a) sample. The offset of the WL-selected clusters is still evident down to these low masses.

\subsection{X-ray morphology of WL-selected clusters}

\subsubsection{The dynamical state of WL-selected clusters}

We wish to investigate the dynamical state of our WL-selected clusters as compared to X-ray-selected cluster samples. We use the centroid shift method of Poole et al. (2006) to determine the dynamical state of the cluster. The centroid shift $(\langle w\rangle)$ was defined as the standard deviation of the distance between the X-ray peak and centroid, measured within a series of circular apertures centred on the X-ray peak and decreasing in steps of 5 per cent from $r_{500}$ to $0.05 r_{500}$. We note that due to the proximity of SLJ1000 to the edge of the field of view (FOV, see Fig. A3), the radius enclosing $r_{500}$ falls outside the ACIS camera. This could bias the measurement of $\langle w\rangle$. To resolve this, a maximum radius of $0.45 r_{500}$ was used, which fell within the FOV. The measured $\langle w\rangle$ increased from $0.105 \pm 0.004$ using the maximum radius of $r_{500}$, to $0.150 \pm 0.007$ using a maximum radius of $0.45 r_{500}$. We note that this does not change the $\mathrm{CC}$ status for SLJ1000. For the remaining clusters where $\langle w\rangle$ could be measured, the radius enclosing $r_{500}$ fell within the detector. The errors on $\langle w\rangle$ were computed from 100 Monte Carlo randomizations of the input image with pixels randomized under a Poisson distribution, therefore taking into account the image quality.

The $\langle w\rangle$ values are given in Table 3. Maughan et al. (2012) used $\langle w\rangle$ to distinguish between 'relaxed' and 'unrelaxed' clusters. For the Maughan et al. (2012) sample of 114 clusters, they adopted a value of $\langle w\rangle=0.006 r_{500}$ below which clusters are classed as 'relaxed', and above which clusters are classed as 'unrelaxed'. Using this threshold value, 25 per cent of the Maughan et al. (2012) sample was classed as 'relaxed'. If we adopt this value for our sample of WL-selected clusters, we find that none of the clusters in Table 3 qualify as 'relaxed'.

The value of $\langle w\rangle$ is somewhat subjective, and the value used to separate relaxed and unrelaxed clusters can depend on the sample of clusters used, and indeed on each individual study. Pratt et al. (2009) used the REXCESS sample of clusters and defined relaxed/unrelaxed clusters using the same method of calculating $\langle w\rangle$ as we employ here. Pratt et al. (2009) found that $\sim 60$ per cent of their clusters has $\langle w\rangle<0.01$, while for our WL clusters we find
Table 3. Table of the derived dynamical properties of the cluster sample.

\begin{tabular}{cccc}
\hline Cluster & $\langle w\rangle\left(10^{-3} r_{500}\right)$ & Cuspiness & $\epsilon_{\mathrm{BCG}}$ \\
\hline SLJ0225.7-0312 & - & - & 0.07 \\
SLJ1647.7+3455 & - & - & $0.15 \pm 0.01$ \\
SLJ0850.5+4512 & $31 \pm 28$ & $0.71_{-0.64}^{+0.44}$ & $0.13 \pm 0.01$ \\
SLJ1000.7+0137 & $105 \pm 4$ & $0.02_{-0.01}^{+0.15}$ & $0.20 \pm 0.02$ \\
SLJ1135.6+3009 & - & - & $0.40 \pm 0.01$ \\
SLJ1204.4-0351 & $28 \pm 13$ & $0.13_{-0.08}^{+0.55}$ & $0.16 \pm 0.02$ \\
SLJ1335.7+3731 & $12 \pm 8$ & $0.24_{-0.17}^{+0.48}$ & $0.11 \pm 0.02$ \\
SLJ1337.7+3800 & $44 \pm 30$ & $0.97_{-0.16}^{+0.19}$ & $0.23 \pm 0.01$ \\
SLJ1602.8+4335 & $7 \pm 4$ & $0.53_{-0.51}^{+0.17}$ & $0.14 \pm 0.02$ \\
SLJ1634.1+5639 & $87 \pm 85$ & - & $0.15 \pm 0.01$ \\
\hline
\end{tabular}

that only one of the seven clusters with measurable $\langle w\rangle$ values are below this threshold. It should be noted that four of the seven clusters in our sample have errors intersecting with the Pratt et al. (2009) threshold; however, both Pratt et al. (2009) and Maughan et al. (2012) do not consider the errors on their classifications. We further note that this comparison is only based on a small number of clusters from the WL.

This result appears analogous to the recent cluster sample from the early release Planck all-sky survey (Planck Collaboration 2011b). These clusters were detected via their SZ signals, and 25 candidates were followed up with snapshot $X M M$ observations. Through a visual inspection of the gas density profiles constructed for each cluster, a large proportion of these SZ-selected clusters appear morphologically disturbed. Since the WL-selected clusters studied in our sample also appear to be morphologically disturbed, selecting clusters from either their SZ or WL signal, as compared to selecting cluster via their X-ray emission, appears to give a more representative distribution of morphologies for clusters.

\subsubsection{The CC fraction of WL-selected clusters}

The absence of relaxed clusters in our sample as indicated by $\langle w\rangle$ suggests we should also see few CC clusters. This also has important implications for cosmology as the $\mathrm{CC}$ fraction and its evolution may be overrepresented/biased in X-ray samples (Santos et al. 2010). This result is not unexpected due to the high X-ray surface brightness at the centre of CC clusters, making them easier to detect in X-ray cluster surveys. Hudson et al. (2010) presented a comprehensive study of $16 \mathrm{CC}$ diagnostics for the HIFLUGCS sample of 64 clusters. They found that, for clusters with low data quality, the cuspiness of the gas density profile is the preferred method to determine the presence of a CC. Cuspiness is defined as the logarithmic slope of the gas density profile at a radius of $0.04 r_{500}$, modelled using the gas density profiles given by equation (1), with the uncertainties derived from the cuspiness values measured from Monte Carlo realizations of the gas density profile. We obtained the cuspiness values from gas density profiles fitted to surface brightness profiles derived from images binned by a factor 2 , with each radial bin containing at least 10 cluster counts. The same fitting process was followed as in Section 3.1 and the cuspiness values were derived from these profiles. Table 3 lists the values of the cuspiness for each cluster where a gas density profile could be obtained.

Maughan et al. (2012) also used the cuspiness to determine the presence of a $\mathrm{CC}$ in their sample of 114 clusters. Above a value of 0.8 , clusters were considered to host a $\mathrm{CC}$, and below they were not. 
If we adopt this value for our sample of six clusters with measurable cuspiness, we find that one of the clusters is considered to host a CC. The Maughan et al. (2012) sample spans a wide range in redshift and data quality, and again the threshold in cuspiness used to define CC/NCC clusters is subjective depending on the sample being studied. The Hudson et al. (2010) sample consists of clusters with very high data quality and the value of the cuspiness is well constrained for all clusters. Hudson et al. (2010) adopted a value for cuspiness of 0.7 to segregate between CC and NCC clusters. If we adopt this value of cuspiness for our sample of clusters, we find only $2 / 6$ of the clusters host a CC. With either criterion, the WL sample contains a smaller percentage of CC clusters than these other samples. We find 16.6 per cent of clusters host a CC when using a cuspiness value of 0.8 , as compared to 26 per cent found in Maughan et al. (2012). We find that 33 per cent of clusters host a $\mathrm{CC}$ when using a cuspiness value of 0.7 , as compared to 55 per cent found in Hudson et al. (2010). This suggests that CC clusters may be overrepresented in X-ray-selected samples of clusters.

\section{DISCUSSION}

In Section 4.2, we showed that WL-selected galaxy clusters are underluminous by a factor $2.1 \pm 0.5$ for a given mass, compared to $\mathrm{X}$-ray-selected clusters. In terms of the mass, we find that the WLselected clusters are overmassive by a factor $1.7 \pm 0.3$ compared to X-ray-selected clusters. The consistency of the LT relation with X-ray-selected clusters (see Fig. 1) suggests that the discrepancy may more likely arise as a result of a bias in the mass. We therefore first investigate the nature of this offset through a study of systematic effects on the WL mass estimates.

\subsection{Possible systematics in the WL masses}

In the following sections, we present a series of plausible effects that could bias the WL mass. These biases are presented as a cumulative effect on the WL mass, and we investigate the effect these biases have on the LM relation. Each correction to the WL mass due to these biases is given in Table 4.

\subsubsection{The effect of cluster centre position on WL Mass}

The X-ray analysis presented in this paper is independent of the WL analysis given in Hamana et al. (2009), and as such, different locations of the cluster centre are used for the two analysis. The WL masses given in Table 2 are derived from fitting NFW models to shear profiles centred on the peak position in the WL mass maps,

Table 4. Table of corrections applied to the WL derived mass for each cluster.

\begin{tabular}{cccc}
\hline \multicolumn{1}{c}{ Cluster } & Centroid & $\begin{array}{l}\text { Correction } \\
\text { Edd. bias }\end{array}$ & Ellipticity \\
\hline SLJ0225.7-0312 & 1.05 & 1.20 & 1.17 \\
SLJ1647.7+3455 & 0.93 & 1.34 & 1.17 \\
SLJ0850.5+4512 & 1.10 & 1.34 & 1.17 \\
SLJ1000.7+0137 & 1.26 & 1.12 & 0.93 \\
SLJ1135.6+3009 & 1.28 & 1.12 & 0.93 \\
SLJ1204.4-0351 & 0.94 & 1.54 & 0.93 \\
SLJ1335.7+3731 & 1.10 & 1.34 & 1.17 \\
SLJ1337.7+3800 & - & 1.28 & 0.93 \\
SLJ1602.8+4335 & 0.98 & 1.18 & 1.17 \\
SLJ1634.1+5639 & 7.42 & 1.70 & 1.17 \\
\hline
\end{tabular}

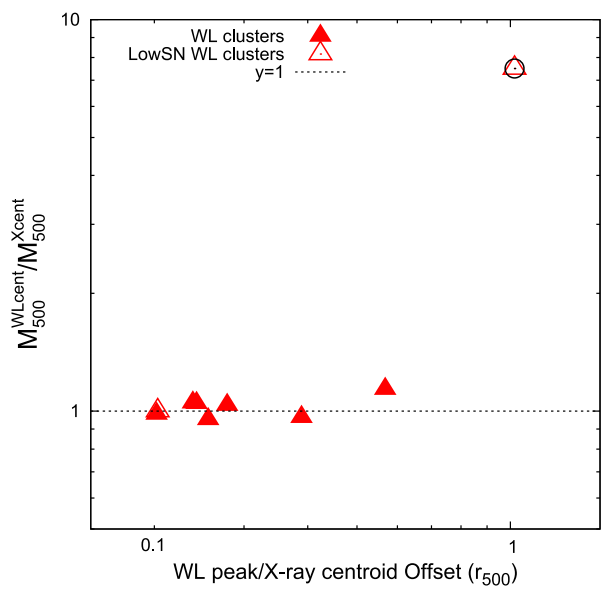

Figure 4. Plot of the ratio of the WL mass derived within the X-ray $r_{500}$ at the WL peak and X-ray centroid, against the offset of the WL peak and $\mathrm{X}$-ray centroid (plotted in units of $r_{500}$ ). The cluster SLJ1634 is highlighted by the black circle.

whereas the luminosities are derived centred on the X-ray centroid. Here, we investigate the effect of deriving the WL masses from profiles centred on the X-ray centroid.

Fig. 4 shows the ratio of the WL mass centred on the peak of the WL signal to the WL mass centred on the X-ray centroid $\left(M_{500}^{\text {WLpeak }} / M_{500}^{\text {Xcent }}\right)$ against the offset of the WL peak and X-ray centroid (in units of $r_{500}$ ). The cluster SLJ1634 is highlighted by the black circle. This cluster shows evidence for a composite structure (see Section 3.3.4) and the WL mass decreases by a factor $\sim 7$ when centred at the X-ray peak. We note for the cluster SLJ1135, the X-ray analysis was centred on the WL peak due to the low SNR of the X-ray data, and no X-ray centroid could be obtained. It is thought that any offset between the WL and X-ray would be minimal since the WL peak is aligned closely with the BCG of the cluster (see Fig. A4b). This figure shows that the ratio of the masses is consistent with a ratio of 1.0 (given by the black dashed line). This result is unsurprising due to the radial range within which the WL masses are derived $(2<\theta<20$ arcmin), to neglect contamination from cluster galaxies. These results are consistent with the results from Okabe et al. (2010a), who find that the relative inaccuracy of cluster centroid position has a negligible effect on the resulting $\chi^{2}$ value of the NFW fit to their distortion profiles, and hence the mass derivation.

The effect of using WL masses centred on the X-ray centroid on the LM relation is shown in Fig. 5 (dashed blue line). The original LM relation from Fig. 3 is given by the dashed green line. By calculating the WL masses centred on the X-ray centroids, the masses have decreased on average by a factor of $\approx 1.1$.

\subsection{2 'Deboosting' the WL mass estimates}

Here, we correct the WL masses by 'deboosting' the shear signal, which is analogous to the effect of Eddington bias (see Section 4.2). Since our sample is based upon a noisy indicator of the cluster mass, the shear signal, clusters with high mass but a low observed shear will not be included in our sample, and conversely clusters with low masses but high observed shear will be included in our sample. Our cluster sample will therefore be biased to clusters with a high shear signal relative to their mass, and due to the slope of the mass function, there will be more low-mass than high-mass clusters close to our detection limit. The overall effect will be to measure 


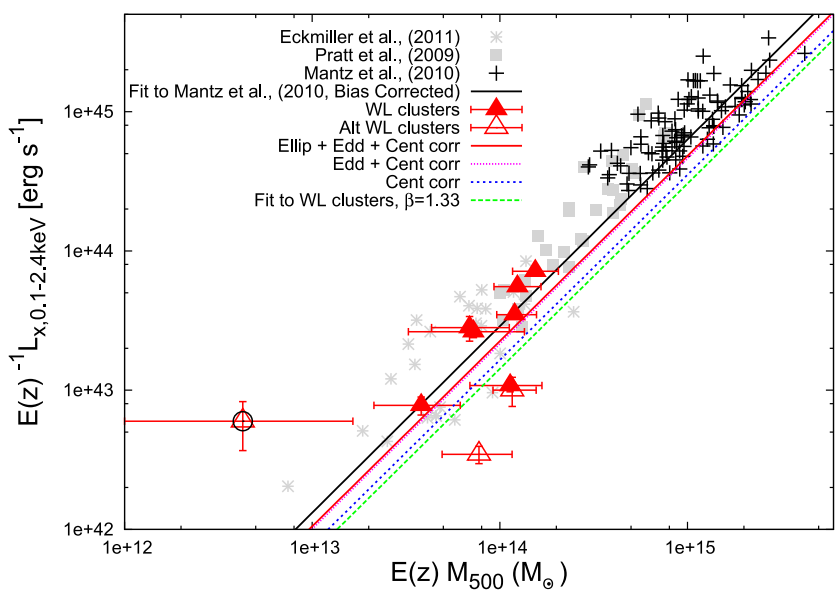

Figure 5. Figure showing the LM relation for the WL-slected clusters, within $r_{500}^{\mathrm{X}}$ (red triangles, clusters analysed using the 'Alternative' method are given by open triangles), with the WL masses corrected for centroid position (Section 5.1.1), Eddington bias (Section 5.1.2), and triaxiality (Section 5.1.3). The cluster SLJ1634 is highlighted by the black circle. A powerlaw fit for the LM relation with the masses corrected for these effects is given by the red solid line. Once again compared to the clusters given in Mantz et al. (2010a, black crosses), and corresponding bias corrected LM relation (black line). The clusters from Pratt et al. (2009) and groups from Eckmiller et al. (2011) are also plotted, but shaded grey for clarity.

high-mass values, due to the high shear signal, and so bias the sample to higher masses. An application of 'deboosting' can be found in Coppin et al. (2006) for a SCUBA source catalogue.

In order to calculate 'deboosted' mass estimates, we follow a similar method to that of Coppin et al. (2006). Initially, the cluster mass is estimated from the shear-derived convergence profile $(\kappa)$ via $\chi^{2}$ fitting with an NFW model. We also have a theoretical prediction of the shear profile for a modified NFW profile. We compute $\chi^{2}$ following the standard procedure from the observed shear profile and the model prediction as a function of halo mass $M$ $\left(\chi^{M}\right)$. From $\chi^{M}$, we compute the best-fitting (non- 'deboosted') WL mass and likelihood interval. Next, we consider $P=\exp \left(-\chi^{2} / 2\right)$ as $P(\kappa, \sigma \mid M)$, and consider the halo mass function as $p(\mathrm{M})$. From Bayes' theorem, we therefore have $P(M \mid \kappa, \sigma) \propto P(M) P(\kappa, \sigma \mid M)$. We then compute $\chi_{\text {deboost }}^{2}=-2 \ln (P(M \mid \kappa, \sigma))$ and find the bestfitting mass and the likelihood function.

This correction decreases the WL mass by an average of $\approx 1.27$. This is shown in Fig. 5 as the dotted pink line, representing the cumulative effect of the centroid position (see Section 5.1.1) and Eddington bias on the LM relation.

\subsubsection{WL biases due to haloes elongated along the LOS}

An additional bias involved in the estimation of WL masses is that due to a triaxial shape of the cold dark matter (CDM) halo (Warren et al. 1992; Jing \& Suto 2002; Shaw et al. 2006). This can lead to errors in the WL masses by $\pm(10-50)$ per cent when spherical symmetry is assumed (Corless \& King 2007; Meneghetti et al. 2010; Becker \& Kravtsov 2011; Bahé, McCarthy \& King 2012). In order to correct for the effect of a triaxial CDM halo, we use the set of simulated clusters studied in Meneghetti et al. (2010). Fig. 17 of Meneghetti et al. (2010) shows the ratio of the weak+strong lensing mass (a non-parametric method combining both strong and WL regimes to calculate cluster mass) to true cluster mass as a function of angle with the projection axis (the angle between the major axis of the cluster inertia ellipsoid and the axis along which the mass distribution is projected). Since our WL masses are based upon NFW profiles, we combine fig. 17 with fig. 16(a) from Meneghetti et al. (2010), to derive the same relation appropriate for NFW profile mass estimates.

It is noted in Marrone et al. (2012) that their plot of fractional deviation in mass from a self-similar $M_{500, \mathrm{wL}}-Y_{\text {sph }}$ as a function of the BCG ellipticity ( $\left.\epsilon_{\mathrm{BCG}}\right)$ looks strikingly similar to fig. 17 presented in Meneghetti et al. (2010). They find that clusters with a BCG ellipticity $\leq 0.15$ have the largest deviation in mass from the self-similar relation. By considering BCGs as prolate systems whose major axis is aligned with the major axis of the CDM halo, circular BCGs indicate the major axis is close to the LOS through the cluster. The viewing angle $(\psi)$ can be calculated as a function of the observed axis ratio $(q)$ and intrinsic BCG axis ratio $(\delta)$, using

$\psi=\arccos \left(\sqrt{\frac{1-(\delta / q)^{2}}{1-\beta^{2}}}\right)$,

where $q=b / a$, and adopting $\delta=0.67$ (Fasano et al. 2010). A $\epsilon_{\mathrm{BCG}}=0.15$ corresponds to a viewing angle of $\psi \simeq 34^{\circ}$, we therefore use clusters below this angle from Meneghetti et al. (2010) to calculate the average of the WL mass to true mass and correct our masses by this factor.

In order to correct our WL masses, we calculate $\epsilon_{\mathrm{BCG}}$ for each cluster from the Subaru images. For clusters which have $\epsilon_{\mathrm{BCG}} \leq 0.15$ (i.e. with a viewing angle $\leq 34^{\circ}$ ), we divide the corresponding WL mass by 1.17 , and clusters with $\epsilon_{\mathrm{BCG}}>0.15$ have their masses divided by 0.93 . The LM relation corrected for triaxiality, Eddington bias, and centroid position is given by the dash-dotted cyan line in Fig. 5.

By calculating $\epsilon_{\mathrm{BCG}}$ for each of our clusters, we have found that 60 per cent appear to be viewed near the LOS, suggesting that WL surveys preferentially select clusters elongated along the LOS. Comparing to Marrone et al. (2012), an X-ray-selected cluster sample, only $\sim 18$ per cent of their clusters have $\epsilon_{\mathrm{BCG}} \leq 0.15$.

\subsubsection{Corrected LM relation}

In Sections 5.1.1, 5.1.2, and 5.1.3, we presented a series of systematic and statistical corrections to our WL cluster masses. We rederive the LM relation for the WL-selected clusters, with the WL masses corrected for these effects. Fig. 5 plots the corrected LM relation, with each of the lines corresponding to a fit with the cumulative effect of these corrections taken into account (see Table 5 for the best fit parameters of each LM relation taking in to account these biases). From the final fit to the mass-corrected LM relation (Fig. 5, red solid line), we find $A_{\mathrm{LM} \text {, corr }}=(5.7 \pm 0.9) \times 10^{43} \mathrm{ergs} \mathrm{s}^{-1}$, with the slope fixed at $\beta=1.33$. The offset from the Mantz et al. (2010a) LM relation has been greatly reduced, and we find that the WLselected clusters are now consistent with X-ray-selected clusters samples, albeit with three clusters being low-luminosity outliers. By considering a series of plausible biases on the WL mass, the discrepancy in the LM relation, when compared to X-ray-selected clusters, can be resolved.

\subsection{Further potential mass biases}

In the following sections, we discuss further biases which may have an effect when comparing WL and X-ray hydrostatic mass calculations. 
Table 5. Observed scaling relations. Each scaling relation is fit with a power-law relation of the form $E(z)^{\alpha} L_{\mathrm{X}}=A_{0}\left(X / X_{0}\right)^{\beta}$, where $X_{0}$ is $2 \mathrm{keV}$ and $2 \times 10^{14} \mathrm{M}_{\odot}$ for $k T$ and $M_{\mathrm{X}}$, respectively.

\begin{tabular}{lcc}
\hline$L_{\mathrm{Xc}}-k T_{\mathrm{c}}$ & $\begin{array}{c}\text { Normalization } \\
A_{\mathrm{LT}_{\mathrm{X}, \mathrm{c}}}\left(10^{43}\right)\end{array}$ & $\begin{array}{c}\text { Slope } \\
B_{\mathrm{LT}, \mathrm{c}}\end{array}$ \\
\hline$L_{\mathrm{X}, \mathrm{c}}-M_{500, \mathrm{WL}}$ & $A_{\mathrm{LM}}\left(10^{43}\right)$ & $2.5 \pm 0.7$ \\
\hline & $3.6 \pm 0.5$ & 1.33 (fixed)
\end{tabular}

Centroid corrected

$\begin{array}{ccc}L_{\mathrm{X}, \mathrm{c}}-M_{500, \mathrm{wL}} & A_{\mathrm{LM}}\left(10^{43}\right) & B_{\mathrm{LM}} \\ & 4.1 \pm 0.6 & 1.33 \text { (fixed) }\end{array}$

Centroid and Edd. bias corrected

$L_{\mathrm{X}, \mathrm{c}}-M_{500, \mathrm{WL}} \quad A_{\mathrm{LM}}\left(10^{43}\right) \quad B_{\mathrm{LM}}$

$5.4 \pm 0.9 \quad 1.33$ (fixed)

Centroid, Edd. Bias, and ellipticity corrected

$L_{\mathrm{X}, \mathrm{c}}-M_{500, \mathrm{WL}} \quad A_{\mathrm{LM}}\left(10^{43}\right) \quad B_{\mathrm{LM}}$

$5.7 \pm 0.9 \quad 1.33$ (fixed)

\subsubsection{WL mass bias due to large-scale structure}

It has been shown that correlated and uncorrelated large-scale structure (LSS) along the LOS, can both induce bias and scatter in the WL mass (e.g. Metzler et al. 1999; Hoekstra 2001; White \& Vale 2004; de Putter \& White 2005; Becker \& Kravtsov 2011; Noh \& Cohn 2011). We expect the effects of LSS and triaxiality to be related, since it can be considered that the major axis of haloes is correlated with filamentary LSS (e.g. Splinter et al. 1997; Faltenbacher et al. 2002; Hopkins, Bahcall \& Bode 2005; Lee \& Evrard 2007; Zhang et al. 2009; Noh \& Cohn 2011). The distribution of BCG ellipticities suggests that 60 per cent of our clusters are viewed with the major axis close to the LOS, from which we can assume that they will also be affected by LSS along the LOS. However, Meneghetti et al. (2010) note that since they consider all the mass within $20 \mathrm{~h}^{-1} \mathrm{Mpc}$ in their lensing simulations, the effect of correlated LSS is partially included in their estimate of the lensing mass. Therefore, the correction applied to our WL masses in Section 5.1.3 based upon Meneghetti et al. (2010), should partially include corrections for LSS. The effect of uncorrelated LSS has been found to not bias the WL mass, but adds additional scatter $\approx 15-30$ per cent as a function of cluster mass (Becker \& Kravtsov 2011; Hoekstra et al. 2011).

\subsubsection{Underestimates of $X$-ray hydrostatic mass}

Simulations have shown that hydrostatic mass estimates of galaxy clusters are systematically biased towards underestimates of the true mass (e.g. Kay et al. 2004; Rasia, Tormen \& Moscardini 2004; Nagai, Vikhlinin \& Kravtsov 2007). Since hydrostatic estimates only take into account thermal pressure, the presence of additional pressure from random gas motions are neglected, and hence bias the mass low. It has been shown that up to $\approx 5-20$ per cent of pressure support comes from random gas motions (e.g. Rasia et al. 2006; Lau, Kravtsov \& Nagai 2009). Since the masses of the Mantz et al. (2010a) clusters are derived from a relation based upon X-ray hydrostatic mass estimates, we can assume that their cluster masses could be underestimated by this amount.

As stated in Section 4.2, the masses in Mantz et al. (2010a) are derived from the gas mass. They note that they incorporate a systematic fractional bias at $r_{500}$ of $0.0325 \pm 0.06$ on the gas mass measurements, based upon the simulations of Nagai et al. (2007). Therefore, this bias will be incorporated in the LM relation comparison. However, as our clusters probe a lower mass distribution than the Mantz et al. (2010a) sample (all $M_{500}>3 \times 10^{14} \mathrm{M}_{\odot}$ ), could the offset be explained by an increasing hydrostatic mass bias for lower cluster masses? The effect would be to not only lower the normalization of the X-ray LM relation, but to also increase the slope, further decreasing the discrepancy of the LM relations. However, our comparison to the lower mass clusters of Pratt et al. (2009) and groups of Eckmiller et al. (2011) do not offer better agreement with our WL-selected clusters. This has also been investigated based on simulations. Jeltema et al. (2008) showed that there is a weak correlation of mass underestimate with cluster mass, with low-mass clusters $\left(\approx 10^{14} \mathrm{M}_{\odot}\right)$ having larger mass bias. Jeltema et al. (2008) find an underestimate of $\sim 20$ per cent at $10^{14} \mathrm{M}_{\odot}$. However, other studies have found no hydrostatic mass bias as a function of mass (e.g. Lau et al. 2009).

\subsection{Are WL-selected clusters underluminous?}

In Section 5.1, we have shown that by considering various systematic effects on the WL cluster mass, we are able to reconcile the differences seen in the LM relation (see Fig. 5). However, our previous work (Hamana et al. 2009, Fig. 5) found that the WL mass is consistent with previous studies when compared to the velocity dispersion $\left(\sigma_{v}\right)$. We reproduce this comparison using $M_{500}^{\mathrm{WL}}$ in Fig. 6. We find that the WL clusters (red triangles) are consistent with the X-ray-selected clusters studied in Hoekstra (2007, grey crosses), in terms of the $M_{500}^{\mathrm{WL}}-\sigma_{v}$ relation. Therefore, we are presented with the situation that the WL mass and $\sigma_{v}$ are consistent and the luminosity and temperature are consistent, implying the properties of the intra-cluster medium (ICM) are inconsistent with $\sigma_{v}$.

To quantify this, we derive a $L_{\mathrm{X} \text {, bol }}-\sigma_{v}$ relation for the WL clusters. Fig. 7 plots the $L_{\mathrm{X}}$, bol $-\sigma_{v}$ relation for our clusters (red triangles), compared to the relation for the HIFLUGCS sample of clusters (cyan diamonds) studied in Zhang et al. (2011). The cluster SLJ1634 is highlighted by the black circle. Due to the composite nature of this cluster (see Section 3.3.4), the value of $\sigma_{v}$ from

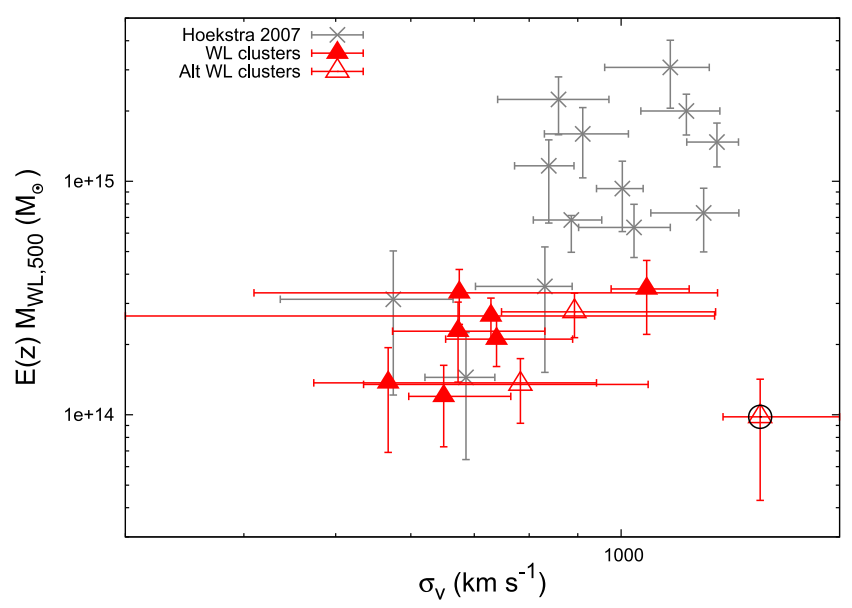

Figure 6. Figure showing the $M_{500}^{\mathrm{WL}}-\sigma_{v}$ relation for the WL-selected clusters (red triangles). Note that the WL masses have not been corrected for the effects discussed in Section 5.1.1, 5.1.2, and 5.1.3. The cluster SLJ1634 is highlighted by the black circle. The clusters studied in Hoekstra (2007) are plotted for comparison (grey crosses). 


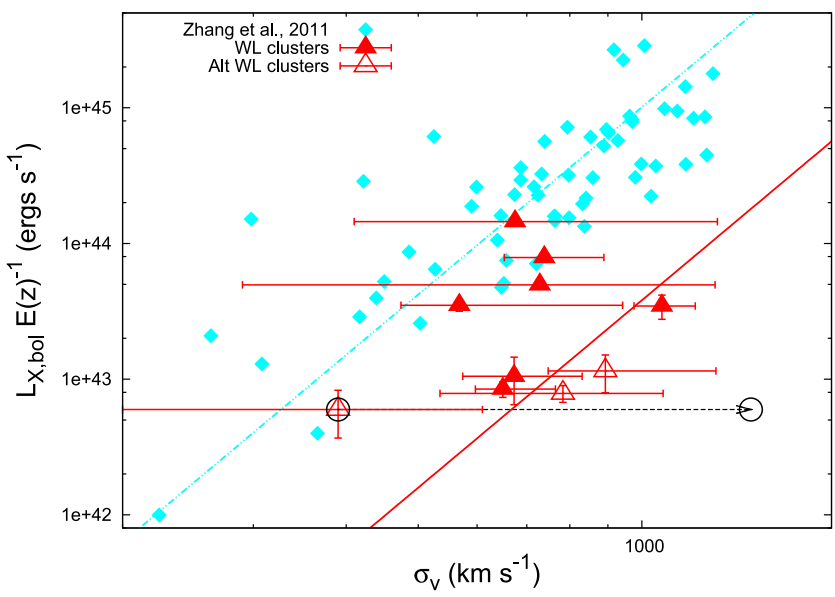

Figure 7. Figure showing the $L_{\mathrm{X}}$, bol $-\sigma_{v}$ for the WL-selected clusters (red triangles). The cluster SLJ1634 is highlighted by the black circle. The open black circle represents the estimate of the velocity dispersion for SLJ1634 from Hamana et al. (2009), when including all spectroscopic galaxies. The HIFLUGCS clusters studied in Zhang et al. (2011, cyan diamonds) are plotted for comparison.

Hamana et al. $\left(2009, \sigma_{v}=1402_{-121}^{+334} \mathrm{~km} \mathrm{~s}^{-1}\right)$ is likely an overestimate. We therefore re-derive $\sigma_{v}$ from our tree analysis using the galaxies from group B (see Fig. A9, black squares), as two of these galaxies are associated with the X-ray emission, finding $\sigma_{v}=390 \pm 220 \mathrm{~km} \mathrm{~s}^{-1}$. We find that five of the WL-selected clusters are clearly underluminous for their velocity dispersion.

These results imply that there is a strong systematic effect remaining that is influencing the WL and dynamical properties, the ICM properties, or both. We consider the most plausible effect to be a high incidence of projected filamentary structure along the LOS to these WL-selected clusters, leading to enhanced WL masses and $\sigma_{v}$ relative to halo mass. From the spectroscopic follow-up, many of the clusters show signs of non-Gaussianity in their velocity dispersion histograms (see fig. 1 in Hamana et al. 2009), which would be consistent with projected filament structure. This however is based on relatively small numbers of spectra in each case (an average of 14 galaxies per cluster), so a more detailed analysis is unable to be performed.

Bower et al. (1997) investigated a sample of optically selected, $\mathrm{X}$-ray underluminous clusters and proposed two scenarios for their observed offset in the $L_{\mathrm{X}}-\sigma_{v}$ relation. Either the clusters have not yet fully formed or are at a stage of rapid mass accretion, which would be manifested in a low X-ray luminosity. The second scenario is that the clusters are embedded in filaments viewed along the LOS, which would lead to an overestimate of $\sigma_{v}$ due to contamination of galaxies along the filament. Other previous studies looking at X-ray underluminous clusters (e.g. Popesso et al. 2007; Balogh et al. 2011; Castellano et al. 2011) favour the scenario that these clusters are still in a stage of formation or that the gas has been expelled. However, since we have measured temperatures for the majority of our clusters and shown that the gas properties scale consistently in the LT relation (and consistently with X-ray-selected cluster samples), we favour the scenario that the WL-selected clusters are embedded in filaments viewed near the LOS. This is further corroborated by our measurements of the BCG ellipticity, where the majority of our cluster BCGs appear circular, implying that the WL selection strongly favours clusters elongated along the LOS and embedded in correlated filaments.

\section{SUMMARY AND CONCLUSIONS}

We have presented the results of X-ray observations of $10 \mathrm{WL}$ selected galaxy clusters in order to determine their X-ray properties. Our main results are as follows.

(i) We find that the scaling relation between X-ray luminosity and temperature is consistent with samples of X-ray-selected clusters.

(ii) We find that the WL-selected clusters are offset from X-rayselected clusters and groups in the LM relation, implying either the X-ray luminosity is underestimated or the WL mass is overestimated.

(iii) The centroid shifts for the WL clusters show that the majority appear dynamically unrelaxed compared to X-ray-selected samples, suggesting X-ray cluster surveys preferentially detect clusters that are morphologically relaxed and/or WL selection favours morphologically disturbed clusters.

(iv) The cuspiness of the gas density profiles shows that two of the clusters in our sample appear to host a CC.

(v) Measuring the ellipticities of the BCGs for each cluster, and assuming that they are intrinsically prolate, we find that 60 per cent of the clusters appear to be viewed with their major axis close to the LOS.

(vi) Through a series of corrections to the WL cluster mass including the centroid position, Eddington bias, and triaxiality, we find the WL-selected clusters are consistent in the LM relation with $\mathrm{X}$-ray-selected clusters.

Our results show that WL-selected clusters are affected by biases which, when combined, have a large effect on the calculated WL mass. We have presented a series of possible biases to explain the large offset of the LM relation when compared to X-ray-selected cluster samples. When considering the cumulative effect of these biases on the WL mass, we reconcile the difference in the LM relation.

\section{ACKNOWLEDGEMENTS}

We thank Massimo Meneghetti for providing data in electronic format of his simulated clusters. PAG also acknowledges support from the UK Science and Technology Facilities Council.

\section{REFERENCES}

Akritas M. G., Bershady M. A., 1996, ApJ, 470, 706

Arnaud M., Pointecouteau E., Pratt G. W., 2007, A\&A, 474, L37

Bahé Y. M., McCarthy I. G., King L. J., 2012, MNRAS, 421, 1073

Balogh M. L., Mazzotta P., Bower R. G., Eke V., Bourdin H., Lu T., Theuns T., 2011, MNRAS, 412, 947

Becker M. R., Kravtsov A. V., 2011, ApJ, 740, 25

Birkinshaw M., 1999, Phys. Rep., 310, 97

Böhringer H. et al., 2004, A\&A, 425, 367

Böhringer H. et al., 2007, A\&A, 469, 363

Bower R. G., Castander F. J., Ellis R. S., Couch W. J., Boehringer H., 1997, MNRAS, 291, 353

Castellano M. et al., 2011, A\&A, 530, A27

Coppin K. et al., 2006, MNRAS, 372, 1621

Corless V. L., King L. J., 2007, MNRAS, 380, 149

de Putter R., White M., 2005, 10, 676

Del Popolo A., Costa V., Lanzafame G., 2010, A\&A, 514, A80

Duffy A. R., Schaye J., Kay S. T., Dalla Vecchia C., 2008, MNRAS, 390, L64

Ebeling H., Edge A. C., Bohringer H., Allen S. W., Crawford C. S., Fabian A. C., Voges W., Huchra J. P., 1998, MNRAS, 301, 881 
Ebeling H., Edge A. C., Mantz A., Barrett E., Henry J. P., Ma C. J., van Speybroeck L., 2010, MNRAS, 407, 83

Eckmiller H. J., Hudson D. S., Reiprich T. H., 2011, A\&A, 535, A105

Faltenbacher A., Gottlöber S., Kerscher M., Müller V., 2002, A\&A, 395, 1

Fasano G. et al., 2010, MNRAS, 404, 1490

Hamana T., Takada M., Yoshida N., 2004, MNRAS, 350, 893

Hamana T., Miyazaki S., Kashikawa N., Ellis R. S., Massey R. J., Refregier A., Taylor J. E., 2009, PASJ, 61, 833

Hoekstra H., 2001, A\&A, 370, 743

Hoekstra H., 2007, MNRAS, 379, 317

Hoekstra H., Hartlap J., Hilbert S., van Uitert E., 2011, MNRAS, 412, 2095

Hoekstra H., Mahdavi A., Babul A., Bildfell C., 2012, MNRAS, 427, 1298

Hopkins P. F., Bahcall N. A., Bode P., 2005, ApJ, 618, 1

Hudson D. S., Mittal R., Reiprich T. H., Nulsen P. E. J., Andernach H., Sarazin C. L., 2010, A\&A, 513, A37

Israel H., Erben T., Reiprich T. H., Vikhlinin A., Sarazin C. L., Schneider P., 2012, A\&A, 546, A79

Jee M. J. et al., 2011, ApJ, 737, 59

Jeltema T. E., Hallman E. J., Burns J. O., Motl P. M., 2008, ApJ, 681, 167

Jing Y. P., Suto Y., 2002, ApJ, 574, 538

Kalberla P. M. W., Burton W. B., Hartmann D., Arnal E. M., Bajaja E., Morras R., Pöppel W. G. L., 2005, A\&A, 440, 775

Kay S. T., Thomas P. A., Jenkins A., Pearce F. R., 2004, MNRAS, 355, 1091

Kravtsov A. V., Vikhlinin A., Nagai D., 2006, ApJ, 650, 128

Lau E. T., Kravtsov A. V., Nagai D., 2009, ApJ, 705, 1129

Lee J., Evrard A. E., 2007, ApJ, 657, 30

Mahdavi A., Hoekstra H., Babul A., Bildfell C., Jeltema T., Henry J. P., 2013, ApJ, 767, 116 (M13)

Mahdavi A., Hoekstra H., Babul A., Bildfell C., Jeltema T., Henry J. P., 2014, ApJ, 794, 175

Mantz A., Allen S. W., Ebeling H., Rapetti D., 2008, MNRAS, 387, 1179

Mantz A., Allen S. W., Rapetti D., Ebeling H., 2010a, MNRAS, 406, 1759

Mantz A., Allen S. W., Ebeling H., Rapetti D., Drlica-Wagner A., 2010b, MNRAS, 406, 1773

Markevitch M. et al., 2003, ApJ, 583, 70

Marrone D. P. et al., 2012, ApJ, 754, 119

Maughan B. J., 2007, ApJ, 668, 772

Maughan B. J., Jones C., Forman W., Van Speybroeck L., 2008, ApJS, 174, 117

Maughan B. J., Giles P. A., Randall S. W., Jones C., Forman W. R., 2012, MNRAS, 421, 1583

Meneghetti M., Rasia E., Merten J., Bellagamba F., Ettori S., Mazzotta P., Dolag K., Marri S., 2010, A\&A, 514, A93
Metzler C. A., White M., Norman M., Loken C., 1999, ApJ, 520, L9

Miyazaki S., Hamana T., Ellis R. S., Kashikawa N., Massey R. J., Taylor J., Refregier A., 2007, ApJ, 669, 714

Nagai D., Vikhlinin A., Kravtsov A. V., 2007, ApJ, 655, 98

Noh Y., Cohn J. D., 2011, MNRAS, 413, 301

Oguri M., Hamana T., 2011, MNRAS, 414, 1851

Okabe N., Takada M., Umetsu K., Futamase T., Smith G. P., 2010a, PASJ, 62,811

Okabe N., Zhang Y.-Y., Finoguenov A., Takada M., Smith G. P., Umetsu K., Futamase T., 2010b, ApJ, 721, 875

Planck Collaboration VIII, 2011a, A\&A, 536, A8

Planck Collaboration IX, 2011b, A\&A, 536, A9

Poole G. B., Fardal M. A., Babul A., McCarthy I. G., Quinn T., Wadsley J., 2006, MNRAS, 373, 881

Popesso P., Biviano A., Böhringer H., Romaniello M., 2007, A\&A, 461, 397

Pratt G. W., Croston J. H., Arnaud M., Böhringer H., 2009, A\&A, 498, 361

Rasia E., Tormen G., Moscardini L., 2004, MNRAS, 351, 237

Rasia E. et al., 2006, MNRAS, 369, 2013

Santos J. S., Tozzi P., Rosati P., Böhringer H., 2010, A\&A, 521, A64

Shan H. et al., 2012, ApJ, 748, 56

Shaw L. D., Weller J., Ostriker J. P., Bode P., 2006, ApJ, 646, 815

Smith R. K., Brickhouse N. S., Liedahl D. A., Raymond J. C., 2001, ApJ, 556, L91

Splinter R. J., Melott A. L., Linn A. M., Buck C., Tinker J., 1997, ApJ, 479, 632

Staniszewski Z. et al., 2009, ApJ, 701, 32

Starikova S. et al., 2014, ApJ, 786, 125

Vanderlinde K. et al., 2010, ApJ, 722, 1180

Vikhlinin A., Kravtsov A., Forman W., Jones C., Markevitch M., Murray S. S., Van Speybroeck L., 2006, ApJ, 640, 691

Vikhlinin A. et al., 2009a, ApJ, 692, 1033

Vikhlinin A. et al., 2009b, ApJ, 692, 1060

Warren M. S., Quinn P. J., Salmon J. K., Zurek W. H., 1992, ApJ, 399, 405

White M., Vale C., 2004, Astropart. Phys., 22, 19

Wittman D., Dell'Antonio I. P., Hughes J. P., Margoniner V. E., Tyson J. A., Cohen J. G., Norman D., 2006, ApJ, 643, 128

Zhang Y., Yang X., Faltenbacher A., Springel V., Lin W., Wang H., 2009, ApJ, 706, 747

Zhang Y.-Y., Andernach H., Caretta C. A., Reiprich T. H., Böhringer H., Puchwein E., Sijacki D., Girardi M., 2011, A\&A, 526, A105 


\section{APPENDIX A: X-RAY AND SUBARU IMAGES OF INDIVIDUAL CLUSTERS}

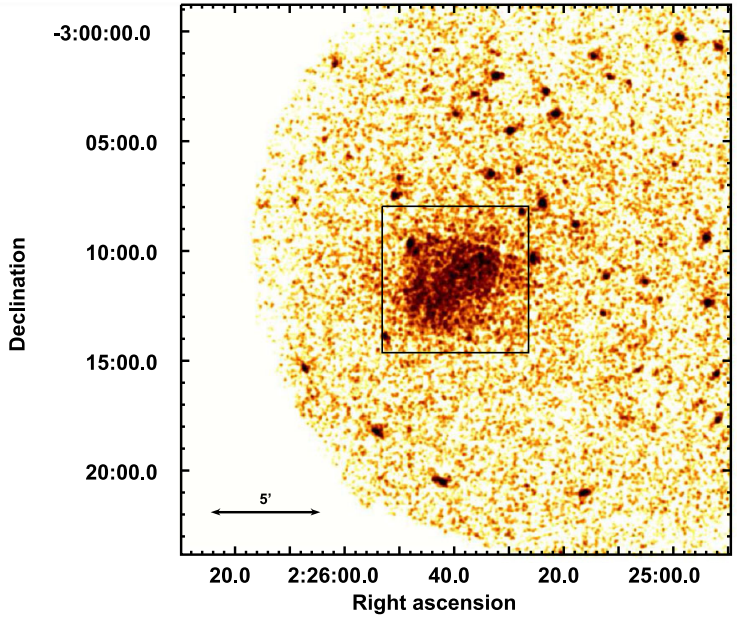

(a)

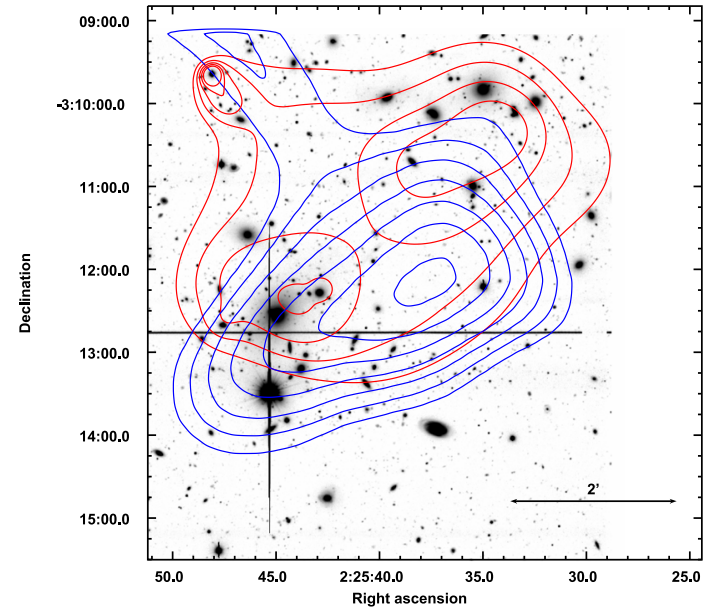

(b)

Figure A1. Images for the cluster SLJ0225 $(z=0.1395)$. Figure (a) shows the XMM image of the cluster in the $0.7-2.0 \mathrm{keV}$ band smoothed by a Gaussian of 1.5 pixel radius (where 1 pixel $=2.2$ arcmin); (b) shows a Subaru image of the cluster within a region 400 arcsec $\times 400$ arcsec in length (shown by the black box in figure a) with X-ray contours (red) and WL mass contours (blue) overplotted. The X-ray contours were constructed from an adaptively smoothed image of the total MOS data, due to the cluster falling on the chip gaps of the pn-camera. The WL contours are taken from Hamana et al. (2009).

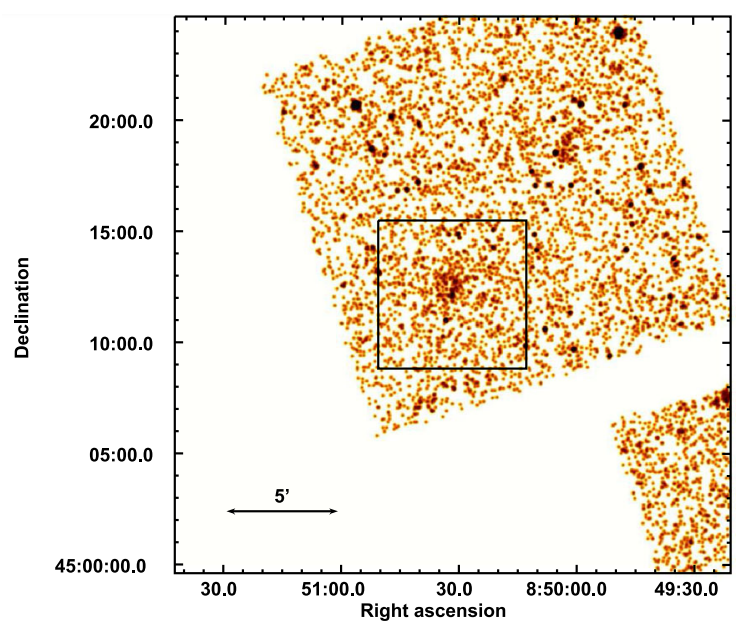

(a)

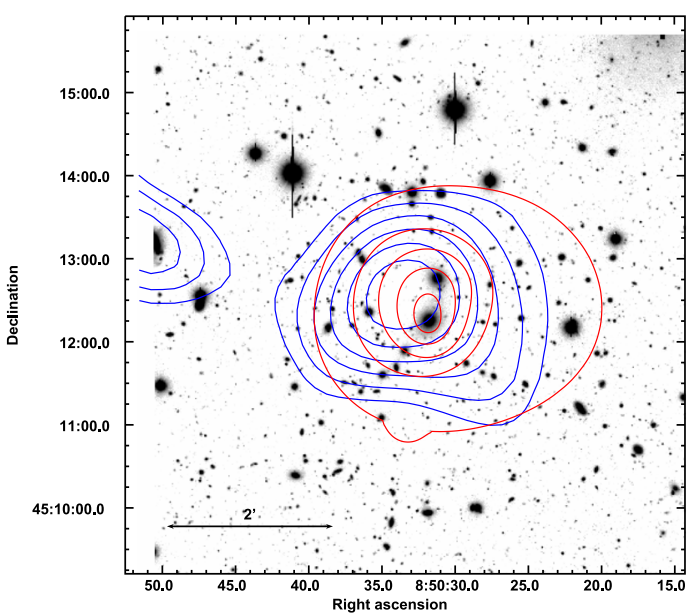

(b)

Figure A2. Images for the cluster SLJ0850 $(z=0.1938)$. (a) shows a Chandra image in the $0.7-2.0 \mathrm{keV}$ band within a region 25 arcmin $\times 25$ arcmin in length, the image is smoothed by a Gaussian of 1.5 pixel radius (where 1 pixel $=1.968$ arcsec); (b) shows a Subaru image of the cluster within a region $400 \operatorname{arcsec} \times 400$ arcsec in length (shown by the black box in figure a) with the X-ray contours (red) and WL mass contours (blue) overplotted. The X-ray contours were constructed from an adaptively smoothed Chandra image of the cluster. 


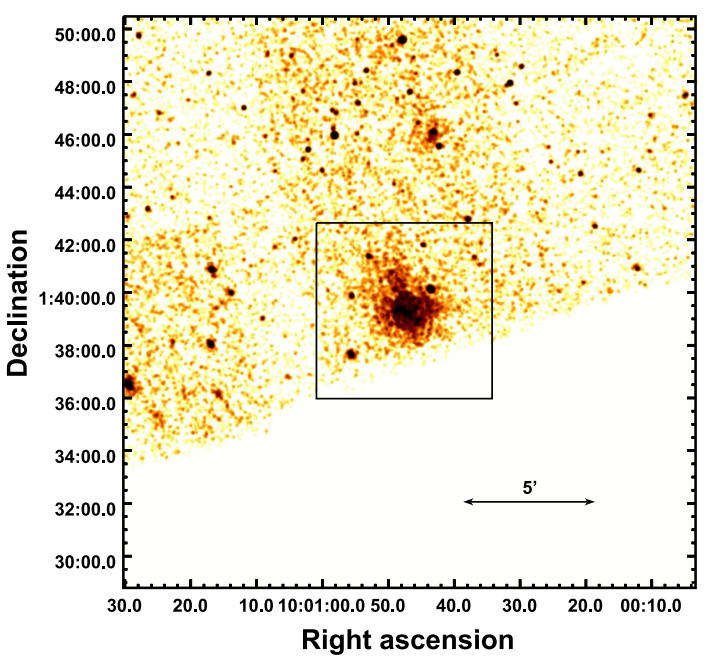

(a)

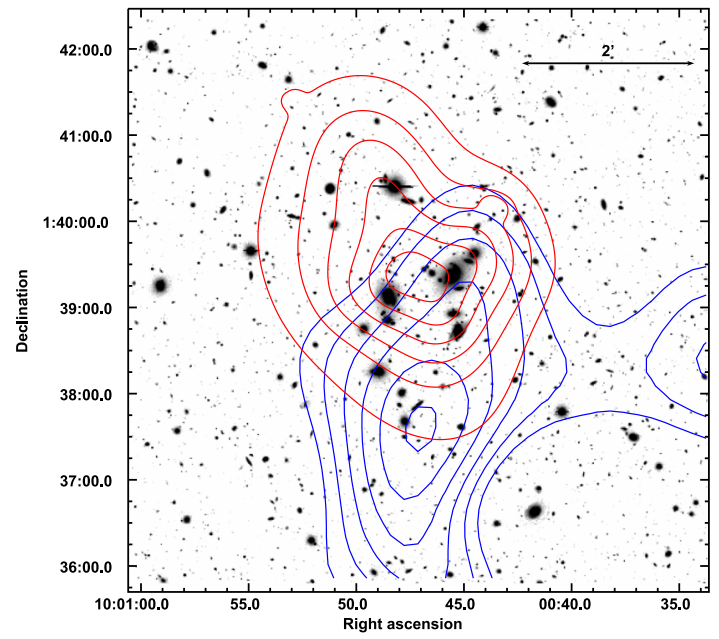

(b)

Figure A3. Images for the cluster SLJ1000 ( $z=0.2166)$. Figures (a) and (b) same as A2.

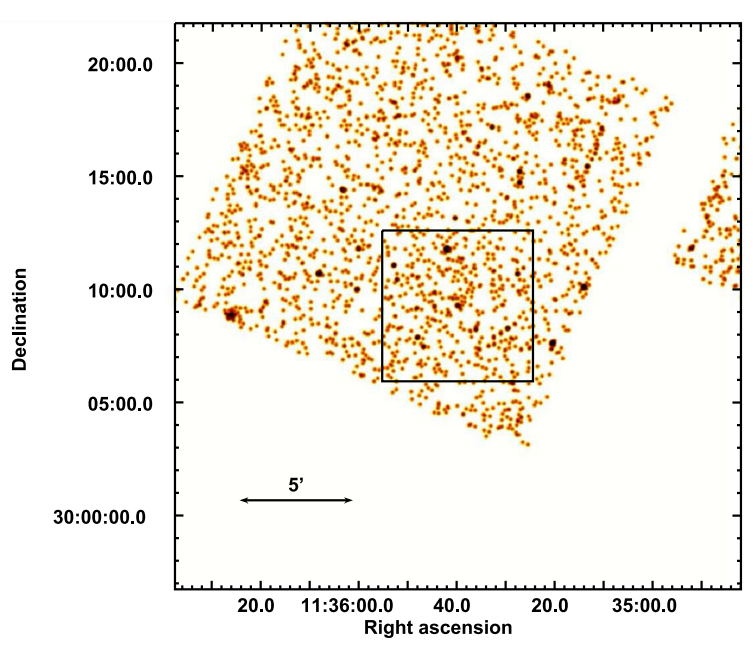

(a)

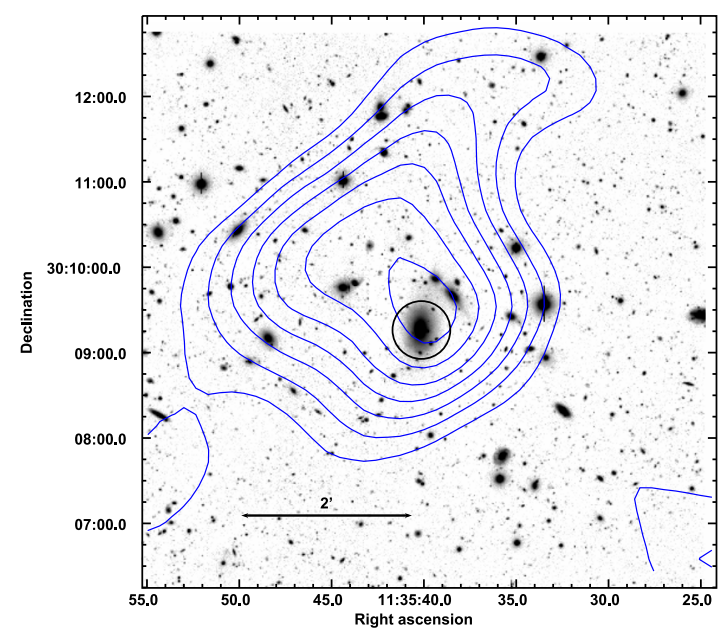

(b)

Figure A4. Images for the cluster SLJ1135 $(z=0.2078)$. Figures (a) and (b) same as A2; however, due to the low SNR of this cluster, an adaptively smoothed image of the cluster was not obtained.

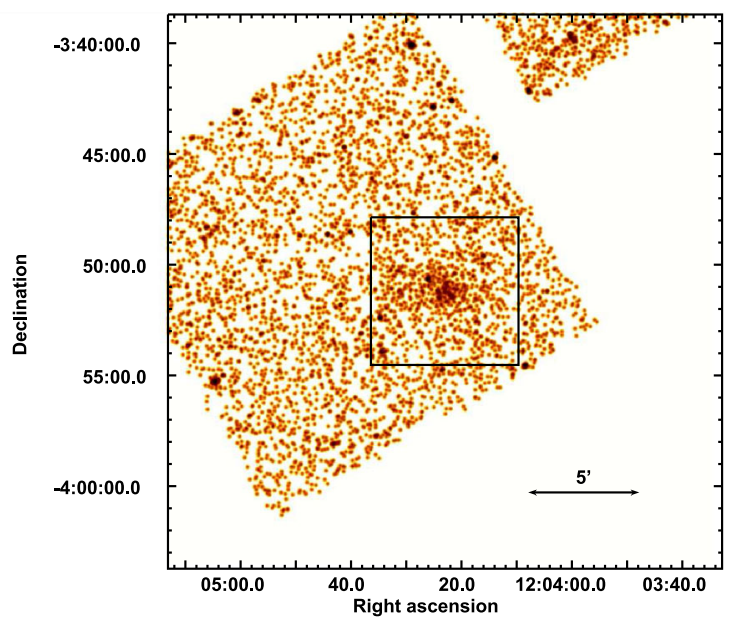

(a)

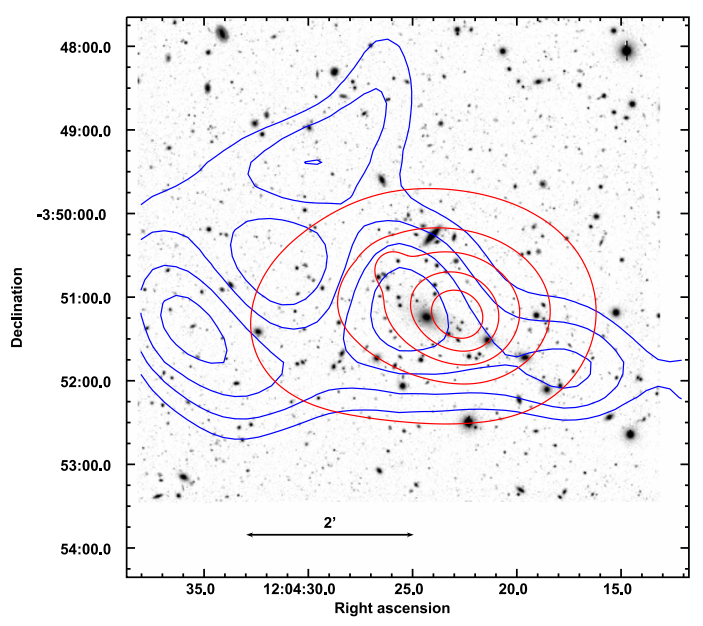

(b)

Figure A5. Images for the cluster SLJ1204 ( $z=0.2609)$. Figures (a) and (b) same as A2. 


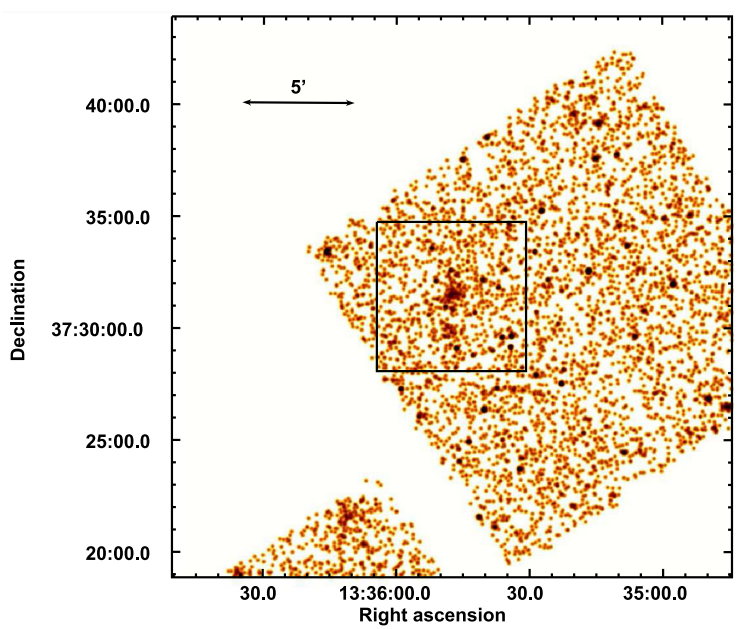

(a)

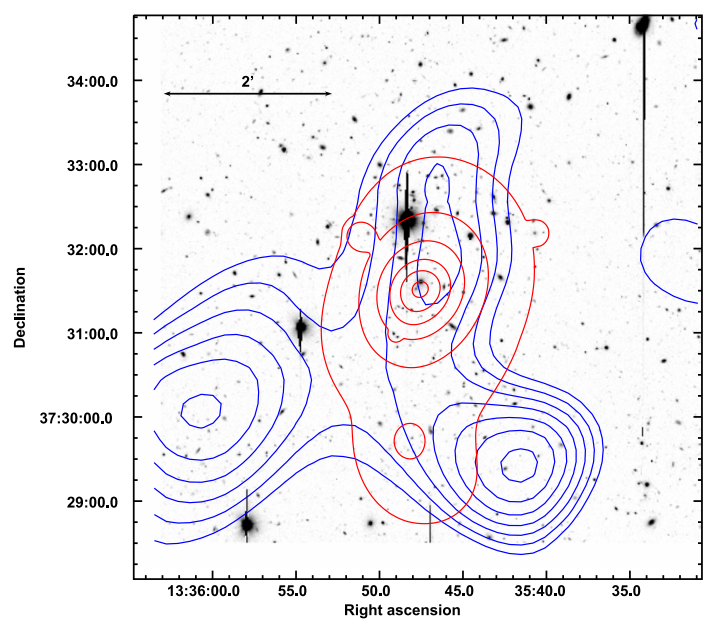

(b)

Figure A6. Images for the cluster SLJ1335 ( $z=0.4070)$. Figures (a) and (b) same as A2.

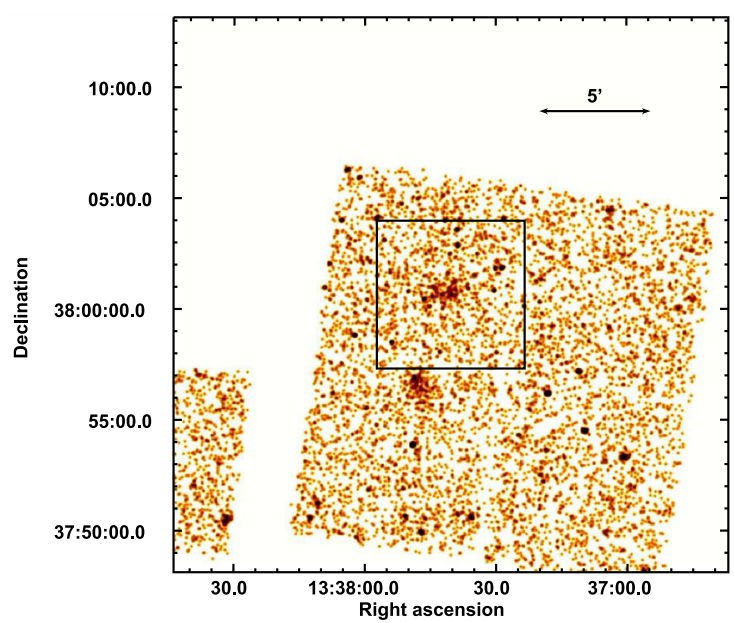

(a)

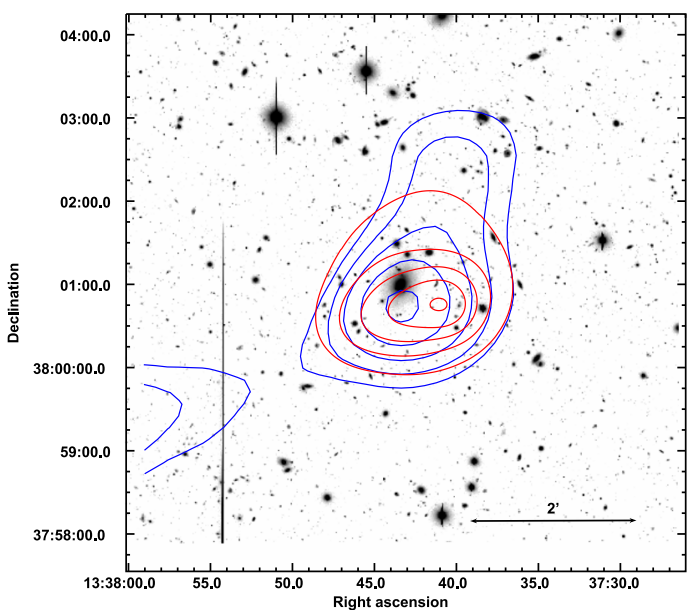

(b)

Figure A7. Images for the cluster SLJ1337 ( $z=0.1798)$. Figures (a) and (b) same as A2.

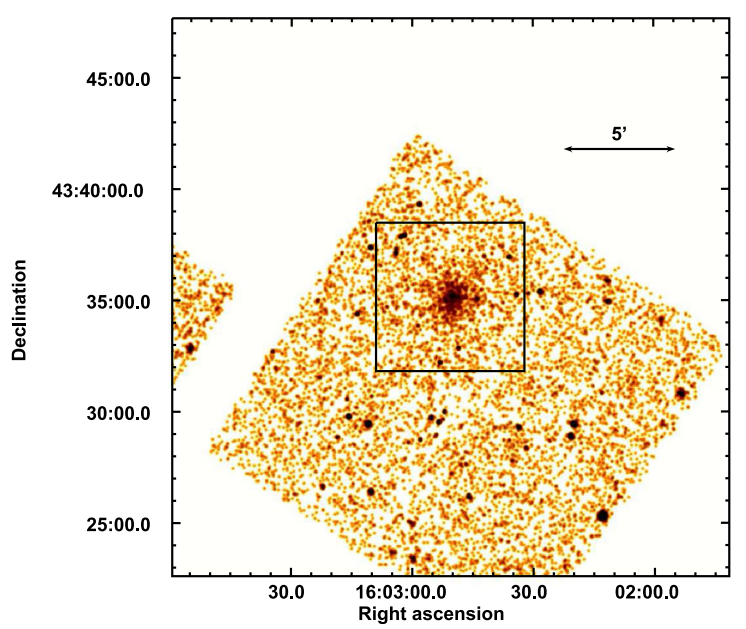

(a)

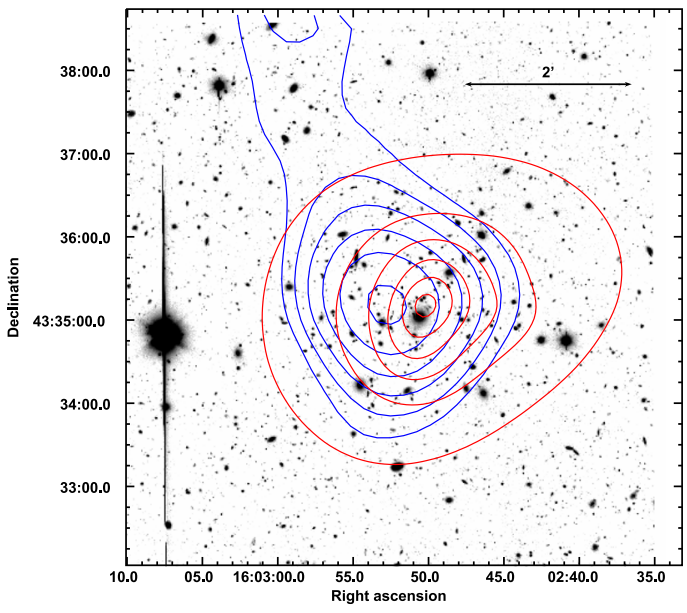

(b)

Figure A8. Images for the cluster SLJ1602 $(z=0.4155)$. Figures (a) and (b) same as A2. 


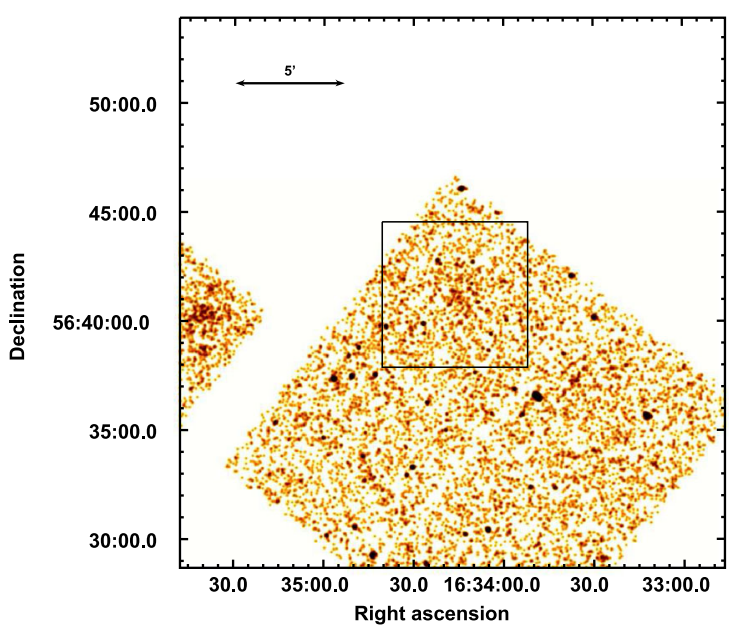

(a)

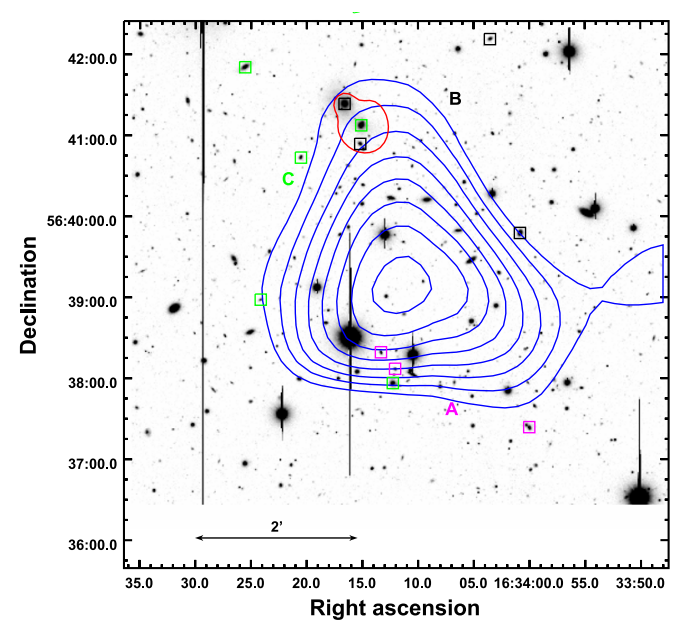

(b)

Figure A9. Images for the cluster SLJ1634 ( $z=0.2377)$. Figures (a) and (b) same as A2. Also highlighted in figure (b) is the locations of spectroscopically confirmed cluster galaxies, separated into three groups A, B, and C (magenta, black, and green squares, respectively) found by our tree analysis (see Section 3.3.4).

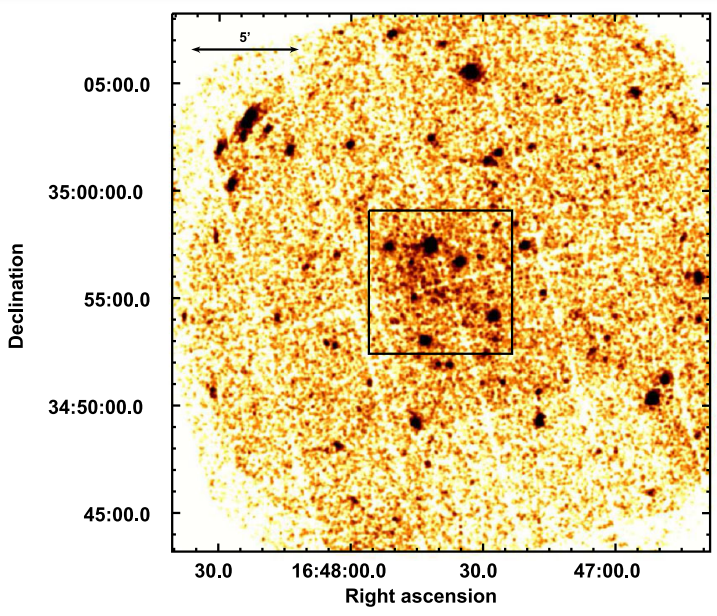

(a)

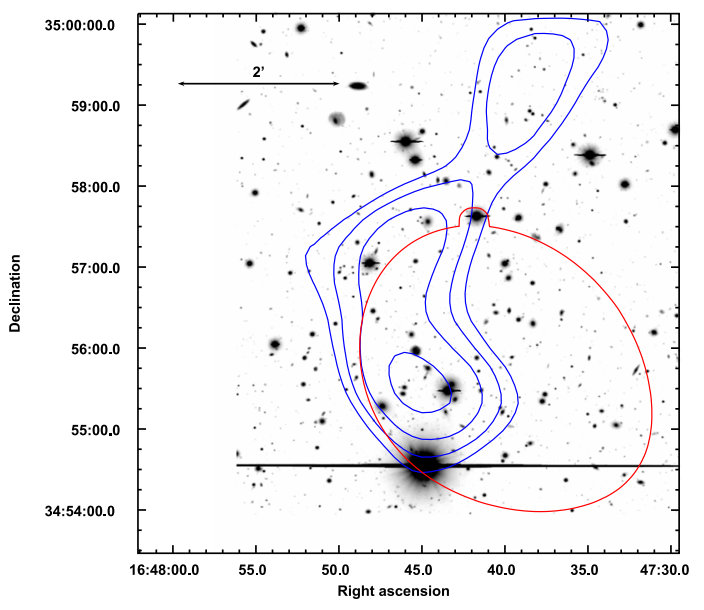

(b)

Figure A10. Images for the cluster SLJ1647 ( $z=0.2592)$. Figures (a) and (b) same as A2.

This paper has been typeset from a $\mathrm{T}_{\mathrm{E}} \mathrm{X} / \mathrm{L} \mathrm{T}_{\mathrm{E}} \mathrm{X}$ file prepared by the author. 